\title{
Des savoirs comme pratiques de problématisation : une approche socio-cognitive en didactique de l'histoire
}

Knowledge as problem-building practices: a socio-cognitive approach in history education

Sylvain Doussot et Anne Vézier

\section{OpenEdition \\ Journals}

Édition électronique

URL : https://journals.openedition.org/educationdidactique/2097

DOI : 10.4000/educationdidactique.2097

ISSN : 2111-4838

Éditeur

Presses universitaires de Rennes

\section{Édition imprimée}

Date de publication : 20 décembre 2014

Pagination : 111-139

ISBN : 978-2-7535-4002-6

ISSN : $1956-3485$

\section{Référence électronique}

Sylvain Doussot et Anne Vézier, « Des savoirs comme pratiques de problématisation : une approche socio-cognitive en didactique de l'histoire », Éducation et didactique [En ligne], 8-3 I décembre 2014, mis en ligne le 20 décembre 2016, consulté le 17 août 2022. URL : http://journals.openedition.org/ educationdidactique/2097 ; DOI : https://doi.org/10.4000/educationdidactique.2097 


\title{
DES SAVOIRS COMME PRATIQUES DE PROBLÉMATISATION : UNE APPROCHE SOCIO-COGNITIVE EN DIDACTIQUE DE L'HISTOIRE
}

Sylvain Doussot, Espé de l'académie de Nantes, université de Nantes, CREN EA 2661.

Anne Vézier, Espé de l'académie de Nantes, université de Nantes, CREN EA 2661.

\begin{abstract}
La mise en évidence de principes structurant les pratiques et les savoirs scientifiques dans le cas des SVT (OrangeRavachol et Beorchia, 2011) ouvre l'analyse didactique en direction des conditions d'accès à un savoir problématisé. Nous nous saisissons de cet outil pour questionner, en histoire, les travaux menés par notre équipe dans le cadre théorique de la problématisation. Par le croisement de réflexions épistémologiques et pratiques en histoire et en didactique, et sur la base d'une séquence forcée (élèves de 10-11 ans), nous identifions ainsi des principes structurants indissociablement théoriques et méthodologiques qui permettent d'étudier simultanément les dimensions cognitives et sociales du savoir en jeu dans la classe. Ce qui prolonge pour notre discipline l'usage d'espaces des contraintes comme outils d'analyse didactique de la logique des savoirs vers la logique sociale de la classe, en vue d'une appréhension des savoirs comme pratiques.
\end{abstract}

Mots-clés : Didactique de l'histoire, problématisation, pratiques de savoir, principes structurants, principes pratiques.

Knowledge as problem-building practices: a socio-cognitive approach in history education

By bringing out principles that structure scientific practices and knowledge (about geology and biology), Orange-Ravachol and Beorchia (2011) lead didactics interpretations towards access conditions to scientific knowledge as knowledge linked to a problem. We seize these structuring principles as tools to question our own work in history education conducted under the theoretical frame of "problematization". About a specifically built course (for pupils of 10-11) and through crossed epistemological and didactical approaches in history and history education, we thus identify inseparable theoretical and methodological structuring principles that give access to both cognitive and social sides of knowledge in the classroom. This extends - for history - the use of "constraint-spaces" as a tool from the analysis of the knowledge logic to the social logic of the classroom aiming at understanding knowledge as practice.

Keywords: History education - problematization - knowledge practices - structuring principles - practical principals. 


\section{INTRODUCTION}

D. Orange-Ravachol et F. Beorchia $\left(2011^{1}\right.$ ) s'intéressent dans un article de la revue Éducation \& Didactique à la notion de principes structurants pour explorer les enjeux épistémologiques et didactiques de différenciation du travail des élèves et des scientifiques pour les SVT. Cette notion caractérise pour les auteures un ensemble de règles qui contrôlent les idées des chercheurs, et régulent leur intégration au corps des savoirs admis dans la communauté disciplinaire. Elle leur permet d'identifier une des difficultés à faire accéder des élèves à des savoirs scientifiques, c'est-à-dire problématisés ${ }^{2}$ : les élèves ne maîtrisent pas ces principes structurants, et ils sont peu mis en jeu en classe.

La fécondité de cette notion épistémologique et didactique nous semble résider autant dans la question de son transfert à notre didactique (de l'histoire) dans une perspective comparatiste, que dans les questions qu'elle peut poser à la didactique en tant qu'approche scientifique. Cette notion nous donne à penser les rapports entre histoire des savoirs (dimension socio-historique), et logique des savoirs (dimension épistémologique), dans une perspective proche de celle des Science Studies (Pestre, 2006). Les principes structurants se sont construits dans le développement historique des communautés scientifiques et mettent en relation textes et pratiques de mise en texte des savoirs. Or cette relation, bien qu'au cœur de l'approche par problématisation - qui repose sur "l'articulation entre textes et pratiques qu'ils organisent et qui les produisent $»$ (Orange \& OrangeRavachol, 2007) - reste difficile à mettre en œuvre lorsqu'il s'agit de référer les disciplines scolaires aux disciplines scientifiques. Si l'apodicticité des textes produits dans la classe est référée aux textes scientifiques (par des analyses a priori qui permettent de dégager les nécessités en jeu dans le problème), les pratiques de mise en texte ne le sont généralement pas. Surtout, les relations entre textes et pratiques de mise en textes relèvent souvent d'une analyse successive - repérage, caractérisation et comparaison des textes produits, puis « remontée » aux pratiques de production - par exemple, cette linéarité est explicite en géologie dans Chalak (2012, p. 126), et en histoire dans Le Marec, Doussot, \& Vézier (2009) - davantage que de l'articulation attendue.

C'est sur la base de cette lecture de la notion de principes structurants que nous avons relu nos études précédentes produites dans le cadre théorique de la problématisation en classe d'histoire : problématisation en référence au cadre théorique développé depuis une vingtaine d'années, notamment dans le séminaire éponyme du CREN (Fabre 2009, Orange 2002). Une première partie (sections 1 et 2 ) croise ces questionnements épistémologiques et pratiques sur un plan théorique, tandis que dans un second temps (sections 3 et 4 ) l'étude est déployée sur la base d'un corpus de données recueilli dans deux classes de CM2.

\section{DES PRINCIPES GÉNÉRATEURS DE PRATIQUES EN CLASSE D'HISTOIRE}

Des pratiques spécifiques à la discipline scolaire instituées en principes admis dans le jeu de la classe

Une première approche des principes qui organisent les pratiques ordinaires en classe d'histoire peut être proposée à travers un corpus exploré ailleurs (Doussot, 2010, 2011). Un travail de groupes (de deux ou trois élèves) en classe de $4^{\mathrm{e}}$ ( $3^{\mathrm{e}}$ année du secondaire) au cours d'une séquence sur la Révolution française requiert la construction d'un récit commun à partir du manuel (pour expliquer la chute de la monarchie en août 1792). Ce travail fondé sur les récits disponibles (ceux des auteurs du manuel, ceux des documents du manuel, ceux des élèves du groupe) s'actualise dans une discussion pour remplir un tableau qui doit lister les explications possibles à la chute de la monarchie. Il révèle, dans les groupes enregistrés, deux principes récurrents de production et de contrôle qui organisent les échanges et la validation des propositions.

Le premier est un principe chronologique qui réfère à l'idée qu'un événement postérieur ne peut expliquer un événement antérieur. Le second est un principe de cohérence des récits avec les modèles de comportement acceptés tacitement : l'enchaînement causal du récit explicatif doit renvoyer à des modèles de comportements admis d'expérience.

Dans ces échanges, le principe chronologique joue le rôle de contrôleur de la proposition d'explication générée par le principe de cohérence avec le modèle de comportement admis. Dans un épisode représentatif, la Terreur semble être considérée comme légitimement attribuée au roi selon le modèle 
du tyran, ce qui pourrait expliquer une réaction violente du peuple ainsi oppressé (la mort du roi). Le modèle de la révolte envers un tyran serait conforme aux conceptions dominantes du roi (Lautier, 1997) et de la révolte. La production de cette explication est ensuite soumise au contrôle de la chronologie par la même élève : la Terreur se termine longtemps après la mort du roi, cette explication est donc impossible. On voit par-là le double processus qui rend compte de l'activité observée dans ce travail autonome : la production de textes de savoir par analogie avec des comportements types, et le contrôle de validité de ces textes par la référence absolue que constitue la chronologie.

Cette illustration donne à voir les effets productifs de ces principes, mais aussi leur nature de principes admis au sein de la classe et de son jeu ${ }^{3}$ ordinaire. Le raisonnement proposé par cette élève à ses camarades n'est pas contesté, pas plus que dans des épisodes identiques du corpus. On propose de parler de principes dans le sens où les élèves s'accordent - même si cela reste tacite - sur des manières d'agir, de parler et de penser en classe d'histoire. Ils ont à voir avec les principes identifiés par Orange-Ravachol et Beorchia (2011), mais ne correspondent pas à ceux des chercheurs.

\section{Des principes pour tenir ensemble pratique et théorie dans une discipline}

Il est intéressant de noter que notre exemple ci-dessus renvoie au travail qu'a effectué Nicole Lautier (1997) sur des notions historiques proches, dans le sens où cette idée de principes générateurs de pratiques disciplinaires gagne bien entendu à être rapprochée des nombreuses études en didactique de l'histoire sur la pensée historienne et son développement chez les élèves ${ }^{4}$. Celles de N. Lautier sont particulièrement pertinentes pour notre questionnement dans la mesure où elles s'inscrivent dans un cadre théorique - celui des représentations sociales (Moscovici) - qui tient la relation entre pensée et pratiques tout le long de l'analyse sans privilégier l'un ou l'autre terme.

À partir d'une enquête menée par entretiens auprès d'élèves de fin de collège et de lycée, elle dégage deux «opérations de conceptualisation » (1997, chap. IV) qui relient les concepts historiques étudiés (monarchie, dictature et démocratie) aux pratiques théoriques des élèves : des opérations de « tâtonnements successifs » et la production d'analogies entre présent et passé, et passé et passé. Si, comme elle le souligne, ces deux opérations ne suffisent pas à épuiser les processus de conceptualisation des élèves en histoire, elles nous semblent essentielles pour appréhender la question des principes sur lesquels s'accordent (généralement de manière tacite) les élèves, et qui structurent leurs activités dans la classe d'histoire. C'est en ce sens qu'elle envisage les « représentations sociales » des élèves comme des « concepts praxéologiques »: des codes qui jouent le rôle de repères pour donner «naturellement » (1997, p. 95) sens à son environnement.

L'appel à ces concepts praxéologiques nous semble une tentative explicite pour rendre compte des relations qu'entretiennent les conceptions sur l'objet étudié (la monarchie, le roi, etc.) et les conceptions sur l'activité (comparer, établir des rapports d'analogie, tâtonner à partir d'un savoir commun pour interpréter un document, etc.). Autrement dit, il s'agit d'une tentative pour dépasser la dualité récurrente entre savoirs et pratiques. Ce dépassement nous semble indispensable dans l'approche didactique des principes qui structurent les pratiques de la discipline histoire. C'est dans cette optique que nous proposons de les nommer « principes pratiques » sur la base d'un usage fait par Bourdieu (1980) dans une perspective plus large, mais dont la valeur heuristique repose précisément sur la volonté de dépasser cette dualité. Pour la didactique de l'histoire, un principe pratique vaut parce qu'il relève de la pratique, même si cette pratique est essentiellement langagière et théorique ${ }^{5}$.

\section{Principes pratiques, principes structurants}

Cette première approche des principes qui structurent les pratiques de la classe d'histoire nous conduit à tenter de les considérer en relation avec les principes structurants tels que Orange-Ravachol et Beorchia les définissent et les utilisent.

Dans leur perspective, les principes structurants organisent les domaines de savoir et permettent une analyse des rapports qu'entretiennent les raisonnements des élèves avec ces principes souvent non explicites. De ce fait, leurs analyses s'ancrent davantage dans le versant épistémologique que dans le versant des pratiques théoriques mises en œuvre 
dans la production des textes de savoir. On peut cependant en faire une lecture davantage croisée. La conclusion de leur article s'y prête particulièrement en soulignant les effets de ces principes lorsqu'ils jouent leur rôle structurant dans les productions savantes.

Ainsi sont-ils d'abord un moyen de faire « barrage contre les irrationalismes » en jouant le rôle de principes de contrôle des récits produits pour formuler des explications. La confrontation des récits possibles à ces principes (actualisme, catastrophisme) permet de sélectionner ceux qui entrent dans une approche scientifique, par contraste avec les « petites histoires » que produisent les élèves. Mais les principes structurants valent aussi sur un autre plan : ils rendent compte des modalités historiques de leur évolution. Sur ce point, la restitution de l'histoire de la naissance de ces principes structurants peut être éclairante, comme les auteures le proposent pour le développement du principe de l'actualisme entre le XIX ${ }^{e}$ siècle et le $\mathrm{XX}^{\mathrm{e}}$ siècle (2011, p. 10-11). Son développement est le résultat des travaux issus de la tension entre deux courants dominants du XIX ${ }^{e}$ siècle pour rendre compte de l'histoire de la Terre (l'uniformitarisme et le catastrophisme).

Pour la science historique, le dépassement des irrationalismes passe de la même manière par des principes qui organisent les pratiques savantes. Ainsi le contrôle des analogies entre passé et présent par les historiens permet de ne pas en rester à une altérité radicale dans laquelle les élèves se trouvent souvent (par exemple, des actes comme la traite et l'esclavage peuvent être expliqués par la nature barbare des hommes de cette époque); mais cela oblige, simultanément, à ne pas assimiler le passé au présent (comme lorsque des élèves expliquent les relations sociales au Moyen Âge par l'application des relations salariales contemporaines - voir Le Marec et al., 2009). Les travaux d'histoire de l'histoire retracent les tensions et leur dépassement à travers la promotion de principes qui structurent des ensembles de travaux (de « programmes » selon les catégories utilisées par Revel, 2009). Ainsi, le programme que l'on peut qualifier de micro-histoire tente de dépasser le dualisme du micro et du macro par un jeu d'échelles systématique (Revel, 1996), qui correspond à la remise en cause du modèle structural dominant jusqu'aux années 1970 (pour la France tout au moins). Par-là, l'histoire de la discipline scientifique permet de resituer l'émergence d'un principe structurant propre à contrôler les analogies et que Ginzburg (1997) formule comme la recherche des rapports entre actions et contextes par opposition avec le déterminisme de l'un ou l'autre terme ${ }^{6}$.

Mais qu'en est-il pour la classe d'histoire? On peut faire l'hypothèse, pour poursuivre l'enquête, que principes structurants au sens de Orange-Ravachol et Beorchia, et principes pratiques au sens utilisé ci-dessus relèvent de la même catégorie qui vise à rendre compte des rapports entre pratiques et savoirs, et qui permettent d'engager des actions sur le savoir. Par conséquent, l'enquête didactique de comparaison entre le monde de la classe d'histoire et le monde des historiens peut se poursuivre si elle s'appuie sur des théories de la pratique susceptibles de dépasser la dualité pratique/théorie.

\section{Rendre compte des incertitudes et des stratégies des élèves}

C'est en ce sens que la notion de " principes pratiques » de Bourdieu (1980) a retenu notre attention. Dans son approche épistémologique des sciences sociales, il distingue explicitement les perspectives phénoménologiques, objectivistes et praxéologiques (1972). Ces dernières sont, selon lui, seules à même de dépasser les limites ethnocentristes des secondes, sans tomber dans les travers subjectivistes des premières. Autrement dit, seule une approche praxéologique peut aider à prendre en charge les biais produits par l'objectivisme qui transforme « l'activité pratique en objet d'observation et d'analyse » (1972, p. 229). Sa spécificité est de s'interroger sur les conséquences épistémologiques et pratiques du retrait opéré de l'urgence et de l'incertitude qui caractérisent la situation pratique. Comment prendre en considération les logiques pratiques des élèves confrontés à la nécessité de choisir et d'agir sans avoir tous les éléments, contrairement à l'observateur qu'est le didacticien ? Le risque est, selon Bourdieu, d'importer dans l'analyse de l'action nos propres principes d'action qui sont, par définition, des principes académiques. Pour notre étude didactique ce risque est décuplé : dans la comparaison entre les deux mondes (savant et scolaire), il y a de fortes chances que nous jugions le monde de la classe par rapport au monde savant des historiens. Or celui-ci ressemble davantage à celui des didacticiens qu'à celui des élèves. D'où la pertinence de cette théorie 
de la pratique qui vise à resituer les actions effectives dans le cadre des actions possibles des acteurs concernés.

Cette perspective est systématisée dans Le sens pratique (1980) avec la notion de " principes pratiques ». Si, comme didacticiens, notre logique d'action est guidée par la construction de savoir, on ne peut faire comme si c'était le cas de la logique d'action des élèves. Le principe pratique est ce qui rend compte du moteur ${ }^{7}$ de l'action dont on n'observe que les effets historiques : les enchaînements d'actions. Ces enchaînements peuvent être représentés sous forme de schémas en patte d'oie, soulignant les nœuds de décision (1980, p. 171). Nœuds qui rendent compte des choix effectifs, mais en relation avec les choix possibles. C'est par la reconstitution du champ des possibles qu'il est envisageable de dégager des principes qui régissent ces choix et qui sont, parce qu'inscrits dans la pratique, rarement explicités par les acteurs. Ces principes renvoient alors davantage à une modalité récurrente d'évaluation des situations qu'à l'application de règles, ce qu'une modélisation objectivante tend à privilégier. C'est le cas dans les principes que nous avons identifiés chez les élèves, comme dans les principes structurants des historiens.

L'important dans ce cadre théorique réside dans cet outil qui consiste à resituer les pratiques dans le champ des pratiques possibles pour éviter l'imposition de nos schémas savants sur des logiques qui en sont éloignées, bien que dans une perspective d'apprentissage théorique (c'est là une difficulté propre à la didactique d'une discipline scientifique). C'est ce qui permet de rendre opérationnels les concepts praxéologiques pointés par Lautier. Le chercheur peut les reconstituer à condition de procéder à la reconstitution de ce champ des possibles à partir de situations singulières (historiques et sociales).

Nous proposons sur cette base de parler désormais indistinctement de principes pratiques pour les élèves et pour les historiens. Cette catégorisation nous permet d'envisager une première piste pour déployer la problématique travaillée par OrangeRavachol et Beorchia. À l'hypothèse des didacticiens d'une opposition entre principes structurants effectifs chez les historiens et absence de principes structurants chez les élèves, pourrait se substituer la présence dans les deux contextes de principes pratiques théoriques, spécifiques au travail sur le passé. Concept médiateur entre ces deux contextes, les principes pratiques rendent possible la comparaison didactique en tenant systématiquement ensemble dans l'analyse les pratiques (de mise en texte) et les savoirs (sous forme de textes) : c'est alors le passage des principes déjà là pour les élèves aux principes élaborés par les historiens au cours du développement de cette science, qui devient central dans notre recherche.

\section{Quelles conditions au passage des principes pratiques de classe aux principes structurants de l'histoire?}

Les différentes études réalisées dans ce sens au sein de l'axe problématisation du CREN depuis quelques années conduisent à identifier un certain nombre de limites à l'adaptation des outils d'analyse développés notamment dans le cadre des sciences de la vie et de la Terre. Au cœur de ces questions pour la problématisation en histoire, l'outil d'analyse que constitue l'espace des contraintes (Orange, 2005) nous a conduits à des modélisations (Le Marec et al., 2009 ; Doussot, 2010, 2011) qu'il convient de remettre en discussion dans la problématique esquissée précédemment du dépassement de la dualité entre social (la classe/la communauté scientifique) et cognitif (l'histoire scolaire/l'histoire scientifique).

Espace des contraintes comme modélisation de la logique cognitive plutôt que sociale

Comme modélisation dans le cadre de la problématisation, l'espace des contraintes vise à éclairer le processus de mise en tension des deux registres propres à rendre compte de l'émergence de conditions de possibilité des explications construites dans la classe (ou chez les savants). Ce qui s'inscrit dans l'idée que le savoir scientifique est spécifique par son apodicticité : il ne s'agit pas simplement d'un « savoir que », mais bien de savoir « pourquoi les choses se sont justement passées ainsi et pas autrement » (Koselleck, 1997 , p. 217) ; le savoir s'incarne dans les raisons que l'on dégage de l'enquête et qui sont valables au-delà du contexte de production.

Dans l'exemple (figure 1), les deux lignes du registre des données et du registre des nécessités (nécessités sur les modèles utilisés par les élèves) 
donnent à voir les éléments présents dans le débat d'une classe de CM1 (9-10 ans) sur les relations entre seigneurs et paysans. Ils mènent à une réponse à la question posée 8 , ce qui relève non pas de la validation du professeur, mais de la mise en évidence de rapports de nécessité entre des éléments apportés par les savoirs de la classe et les idées des élèves. Autrement dit, l'espace des contraintes est ici un outil pour l'analyse a posteriori des processus de construction de raisons qui permettent d'éviter le court-circuit de la validation par le maître.

Dans cet exemple, si la nécessité d'ordre militaire d'un château fort dans la société féodale fait partie $\mathrm{du}$ registre des explications initiales des élèves (les seigneurs se battent entre eux, les paysans y trouvent refuge), elle constitue en même temps un obstacle au passage à une explication prenant en compte les nécessités économiques et sociales (le château est au centre de la seigneurie considérée comme « exploitation agricole » : l'échange de terres contre des récoltes et du travail sous forme de corvées, dont l'entretien du château) ; passage qui n'est pas dissociable d'un rééquilibrage des relations entre seigneurs et paysans, le premier ayant besoin du produit du travail des paysans pour affirmer sa puissance militaire (entretenir un nombre important de combattants).

Par cette mise en tension des données et des modèles, les explications d'un rapport de coercition et d'un rapport salarial importé du présent, au début de la séquence, sont identifiées comme insuffisantes, et dépassées par l'émergence, dans certains énoncés d'élèves, de la nécessité d'un équilibrage différent des relations socio-économiques.
La logique du savoir est bien rendue dans cette modélisation, qui renvoie aux savoirs de référence. Les différentes configurations qui ont marqué l'historiographie de cette période rendent compte notamment d'une émergence d'explications socio-économiques en complément aux explications d'ordre militaire.

Pourtant, le processus de modélisation des chercheurs passe par des choix qui laissent en suspens la logique des élèves : quel sens donnentils à l'émergence de ces nécessités observées par les didacticiens ? Tous les élèves n'ont sans doute pas construit le même sens à la discussion. Les choix opérés dans les tours de parole pour produire ce modèle rendent compte de la logique savante des savoirs, mais ignorent la logique sociale du groupe classe. L'ensemble des choix énonciatifs possibles des élèves, et donc leurs incertitudes, ne sont pas pris en compte.

Cet écart est d'autant plus intéressant dans notre problématique qu'il renvoie à ce que Fabre (2009) souligne de la discontinuité fondamentale du temps de la recherche identifiée par Bachelard (1949, p. 86-97) contre la continuité du temps logique reconstruit après coup ${ }^{9}$. L'historien s'adresse à ses contemporains qui savent la science actuelle, comme le didacticien ne peut négliger le savoir scientifique le plus récent pour penser l'apprentissage. Par conséquent, c'est bien le travail de l'écart entre la logique, fondée sur les connaissances actuelles, et les efforts - historiques des scientifiques ou actuels de la classe - incrustés dans les pratiques et leur temps propre, qui vaut pour l'analyse (historique ou didac-

\begin{tabular}{|l|l|l|l|l|}
\hline $\begin{array}{l}\text { Registre des } \\
\text { données }\end{array}$ & $\begin{array}{l}\text { Un autre seigneur peut } \\
\text { attaquer }\end{array}$ & $\begin{array}{l}\text { Le seigneur a les moyens de se } \\
\text { défendre }\end{array}$ & Les paysans seuls ne peuvent se défendre \\
\hline $\begin{array}{l}\text { Registre des } \\
\text { nécessités }\end{array}$ & $\begin{array}{l}\text { Nécessité d'un lieu } \\
\text { défensif }\end{array}$ & $\begin{array}{l}\text { Le travail des } \\
\text { paysans est } \\
\text { nécessaire }\end{array}$ & $\begin{array}{l}\text { Nécessité de proté- } \\
\text { ger les paysans }\end{array}$ & Nécessité de construire et d'entretenir le château \\
\hline $\begin{array}{l}\text { Registre des } \\
\text { explications }\end{array}$ & Le château fort comme forteresse et comme centre de la seigneurie banale \\
\hline
\end{tabular}

Figure 1. Espace des contraintes (Le Marec et al., 2009, p. 15)

On voit dans cette modélisation que les nécessités acceptées initialement s'enrichissent de nécessités qui n'ont de sens que dans le cadre des relations économiques et sociales de l'époque. C'est ce qui rend raison du paradoxe porté par la question fil rouge de la séquence (accepter de construire et d'entretenir le château). tique), mais qui n'est pas pris en charge dans cette modélisation de l'espace des contraintes.

L'espace des contraintes renvoie de ce point de vue à la «science détemporalisante » évoquée par Bourdieu (1980), ce qui réduit fortement l'appréhension du temps de la recherche, ou tout au moins la manière dont il est vécu par les acteurs de la classe. 
Dès lors, la discontinuité fondamentale de la rupture, des obstacles et du problème - la triangulation essentielle de l'épistémologie bachelardienne (Fabre, 2009) - a-t-elle un sens pour les élèves ? La rupture identifiée par les chercheurs est-elle différente, du point de vue des élèves, de la succession habituelle des savoirs propositionnels de la classe d'histoire?

\section{Une absence de prise en compte des logiques sociales}

Ce type de questions nous semble faire écho aux tentatives de rapprochement entre le cadre de la problématisation dans son inspiration initiale (bachelardienne et poppérienne notamment), et le cadre socio-historique d'analyse des pratiques langagières (Jaubert, Rebière \& Bernié, 2003 ; Jaubert, 2007).

En analysant les processus qui conduisent à des modifications des manières d'agir-parler-penser dans la classe, en référence aux manières d'agir-parlerpenser chez les savants, le cadre socio-historique vise à rendre compte des pratiques qui permettent de gérer l'hétérogénéité des contextes de savoir dans la classe. Ce rapprochement entre les deux cadres théoriques repose notamment sur les dimensions argumentative et dialogique de la construction de savoirs scientifiques, processus dans lequel les pratiques langagières, sans être exclusives, jouent un rôle essentiel. Comme le souligne Orange (2005, p. 81), « la formation de la problématisation ne peut pas être simplement considérée comme une traduction d'une problématisation qui existerait déjà, sous forme implicite, dans la "tête" du chercheur. Le prouve tout le travail de pensée lié à la verbalisation, attesté aussi bien par ce qu'en disent les chercheurs eux-mêmes que par les psycholinguistes de la tradition vygotskienne. La problématisation scientifique est donc nécessairement liée à un travail langagier ; celui-ci n'en est pas qu'un épiphénomène ».

Si l'on remet en jeu notre exemple des relations entre seigneurs et paysans, les formulations identifiées par les chercheurs comme essentielles dans le processus de rupture relèvent ainsi de contextes multiples qu'on agrège dans l'espace des contraintes. Les interventions des élèves dans le débat final relèvent parfois du savoir construit juste avant dans un contexte strictement scolaire, parfois des conceptions initialement travaillées. Au sein de cette hétérogénéité des contextes d'énonciation dans la classe, la condition soulevée par un élève pour le seigneur de ne pas interdire l'usage du pressoir à tous les paysans au risque sinon de tarir la production destinée au seigneur, est loin d'être formulée comme une montée en généralité à propos des relations au sein de la seigneurie (au-delà du cas des documents travaillés), encore moins comme un gain d'universalité (au-delà de l'ici et maintenant de la discussion de cette classe), pour reprendre les critères de mise en rapport des deux communautés mis en exergue par OrangeRavachol et Beorchia (2011). Elle rend seulement compte d'un gain en apodicticité ${ }^{10}$ : ménager les paysans pour le seigneur est bien une condition de possibilité de leurs rapports, qui est mise au jour par cet élève et qui conteste le savoir déjà là d'un rapport très déséquilibré en faveur du seigneur. C'est bien en quoi la modélisation par l'espace des contraintes se centre sur l'aspect le plus épistémologique du processus de problématisation, la logique des savoirs entre eux (apodictiques et non propositionnels); et non sur les dimensions socio-cognitives que soustendent généralisation et universalisation (au-delà du travail de la classe).

C'est en ce sens que ce corpus a été retravaillé par la mise en tension du cadre théorique de la problématisation et celui de l'approche socio-historique des pratiques langagières (Doussot, 2011). Nous avons pu dégager quelques pistes dans la perspective de la prise en compte de la logique sociale des acteurs de la classe, en relation avec la logique épistémologique de l'espace des contraintes.

En particulier, les outils graphiques que constituent les listes utilisées au cours de la séance de débat jouent un rôle d'intégration socio-cognitive des savoirs en jeu, comme système sémiotique en débat. Les élèves préparent sous forme de listes individuelles les réponses à la question initiale reposée en séance 5, sous la condition de s'appuyer sur le travail des quatre premières séances. Puis la discussion générale est elle-même appuyée graphiquement par l'inscription progressive au tableau par le maître des éléments stabilisés progressivement par la classe. La liste orale qui structure la discussion (voir schématisation : Doussot, 2011, p. 178-179), reprise au tableau donne à voir à tous sa dimension cumulative : le dernier élément inscrit au tableau (le seigneur a besoin des paysans) n'a pas de sens sans les éléments précédents qui réfèrent au travail de la classe (par exemple : le seigneur peut les faire partir des tenures). 
L'important dans l'analyse de ce processus réside dans la prise en compte progressive des activités précédentes qui fondent localement les conclusions ainsi inscrites - prise en compte qui modifie non seulement le texte du savoir (les relations sont moins déséquilibrées qu'on ne le pensait) mais simultanément les positions énonciatives des élèves et du maître. Dans la situation de ce débat, les conclusions inscrites au fil des échanges rendent compte d'énonciations d'élèves qui valent par la validation par le maître (c'est lui qui inscrit), mais aussi par la validation sur la base du travail des conceptions des élèves (séances 1 et 2) et des savoirs transmis sur la période (séances 3 et 4). Dans ce processus cumulatif porté par les écrits intermédiaires sous forme de listes, l'objet de la discussion passe des hypothèses aux raisons de choisir telle ou telle hypothèse.

Ainsi cette "situation forcée " (Orange, 2010) donne à voir le rôle central que peuvent jouer dans l'analyse les systèmes sémiotiques spécifiques appuyés sur des instruments. Ici les listes successives objectivent non seulement les textes stabilisant le savoir local, mais également sa validation collective. Ce sont ces systèmes qui rendent potentiellement compte des liens entre nécessités dans la logique du savoir (nécessité d'un intérêt pour le seigneur de ménager ses paysans) et nécessités dans la logique sociale (nécessité d'un accord sur les critères de validation). Ce sont ces dernières dont la modélisation par l'espace des contraintes ne rend pas compte, s'en tenant aux nécessités épistémiques analysées a posteriori.

\section{Une hypothèse soutenue par les travaux en sciences de la science}

L'hypothèse d'une articulation de nécessités épistémiques et sociales ancrées dans des systèmes sémiotiques spécifiques prend appui dans le champ de recherche des Science Studies (Pestre, 2006 ; Shinn et Ragouet, P., 2005) qui pose depuis plusieurs décennies des questions fécondes pour comprendre les sciences, leur développement et leur apprentissage. Elles remettent en particulier sur le métier la séparation entre philosophie des sciences, sociologie et histoire des sciences : autant de catégories que les didactiques se doivent de questionner. Et notamment lorsqu'elles s'appuient, comme le fait le cadre théorique de la problématisation, sur les trois piliers suivant : la philosophie (Fabre, 2009), une didactique fortement liée à l'épistémologie des sciences (Orange, 2005), et l'approche socio-historique en psychologie (Jaubert, 2007).

En effet, ces différentes approches des sciences « en action» (Latour, 1989) réfèrent à des points de vue variables souvent opposés. Entre la logique des savoirs prise comme engendrant par elle-même la production de nécessités à un extrême, et la logique sociale comme production de savoirs par mise en concurrence à l'autre extrême, comment choisir ? Et comment articuler, à l'intérieur même de la « logique sociale », une division entre des approches de la « communauté » scientifique (dans une approche mertonienne classique de la sociologie, dont les sujets seraient mus par l'autonomie et le désintérêt) et des approches qui dénient la spécificité du laboratoire comme lieu principal de la science (incarnées en particulier par B. Latour, pour qui les sujets seraient mus par l'intérêt particulier, voire le cynisme)? L'enjeu du dépassement de la dichotomie logique des savoirs/logique sociale est important : il s'agit de rendre compte du moteur de la problématisation, et par-là des conditions à l'intervention didactique.

L'histoire de l'évolution des principes structurants que proposent Orange-Ravachol et Beorchia dans leur article, rend compte d'une dynamique épistémologique qui fait naître des tensions entre principes, leur propre dépassement. Histoire d'inspiration explicitement poppérienne chez les auteures, qui tend à exclure « le monde des sujets » au profit d'un monde peuplé de systèmes théoriques. Mais l'inclusion de ces principes dans la catégorie bourdieusienne des principes pratiques que nous proposons fournit des outils conceptuels pour articuler cette histoire des idées à une histoire sociale de la science.

\section{Dans des pratiques sociales (non scientifiques)}

L'appréhension savante de la pratique et de ses incertitudes passe par la prise en compte de la représentation du réel par les agents, représentation qu'une perspective objectiviste tend à récuser, faisant des agents des exécutants inconscients de règles qui les dépassent. Mais substituer « la dialectique des stratégies à la mécanique du modèle " ne doit pas faire " retomber dans l'anthropologie imaginaire des théories de l'"acteur rationnel" » (Bourdieu, 1980, p. 170) : la récusation du structuralisme levi-straus- 
sien n'est pas la négation de l'existence de structures (d'habitudes, de cultures, etc. selon les auteurs), mais celle de l'inconscience de ces structures. La prise en compte des représentations du réel des agents est donc la condition pour établir des principes pratiques comme « modèles générateurs » de l'activité, c'està-dire cherchant à rendre compte d'un sens du jeu qui, lui, constitue le moteur même de l'activité. Dans ce but, il est indispensable pour l'observateur de construire le champ des possibles des acteurs en situation, autrement dit, si l'on suit J. Bazin qui sur ce plan poursuit de manière critique et argumentée les propositions de Bourdieu, " pour apprendre comment ils agissent, je dois entreprendre de noter patiemment, au coup par coup, ce que font ces gens dans telle et telle circonstance, et donc aussi ce qu'ils pourraient faire mais qu'ils ne font pas ou du moins pas habituellement ${ }^{11}$ " (2008, p. 355-356). Par cet artéfact il s'agit de rendre compte de la logique des pratiques en situant les acteurs les uns par rapport aux autres mais en relation avec les enjeux qui les animent. D'où la pertinence de la notion de jeu social pour décrire cette construction, jeu dans lequel les différenciations relèvent toujours à la fois du social et de la nature de l'enjeu. Dans ce cadre, les structures - des régularités plutôt que des règles - prennent en compte notamment les « coups » légitimes pour les différents joueurs/acteurs, quand bien même ce qu'il est ainsi « convenable » de faire ne constitue pas la totalité de ce qui se fait ou peut se faire : cette distinction est indispensable pour ne pas exclure la logique de la pratique (de classe), de la pratique de la logique (des didacticiens).

\section{Dans les pratiques scientifiques}

Ces perspectives se spécifient en s'attachant à des pratiques théoriques. La " matrice pratique " que constitue le sens du jeu "produire des savoirs » vaut par relation dialectique avec la « matrice théorique » que constituent les principes pratiques qui permettent «d'engendrer sur le papier » ce champ des pratiques de savoir possibles. On voit que les choses se compliquent puisque l'activité scientifique repose précisément sur l'ambition de maîtrise rationnelle de ses propres pratiques.

Ce qui distingue en effet les pratiques scientifiques c'est que les principes qui les guident sont un enjeu propre aux participants au champ ${ }^{12}$. Parmi ces pratiques, l'évaluation des textes soumis pour publication se fait en référence explicite aux principes structurants de la discipline considérée, qu'ils relèvent des cadres théoriques, des concepts ou des méthodes. En conséquence, la construction du champ d'un domaine scientifique a la particularité de rendre compte des pratiques habituelles en y incluant les pratiques d'évaluation de ces pratiques. Autrement dit, la différenciation sociale au sein d'un champ scientifique - les positions et dispositions des agents dans le champ - s'établit sur un double plan cognitif et social. Comme le formule Bourdieu (2001, p. 151):

« Substituer au rapport entre un sujet (le savant) et un objet, un rapport entre sujets (l'ensemble des agents engagés dans le champ) à propos du rapport entre le sujet (le savant) et son objet, conduit à rejeter à la fois et la vision réaliste naïve selon laquelle le discours scientifique est un reflet direct de la réalité, pur enregistrement, et la vision constructiviste relativiste, selon laquelle le discours scientifique est le produit d'une construction, orientée par des intérêts et des structures cognitives, qui produiraient des visions multiples, sous-déterminées par le monde, de ce monde [...]. La science est une construction qui fait émerger une découverte irréductible à la construction et aux conditions sociales qui l'ont rendue possible. »

C'est par cette spécificité des pratiques scientifiques qu'il est, selon Bourdieu, possible d'élargir et de compléter la vision bachelardienne du travail scientifique selon laquelle « le fait scientifique est conquis, construit, constaté » (2001, p. 143). En particulier, l'histoire des sciences, au moins depuis Kuhn (1990), fait de la tension entre science ordinaire et innovation - « tradition et changement dans les sciences » dit le sous-titre de son ouvrage ${ }^{13}-$ le cœur de la dynamique d'accumulation des savoirs scientifiques. Elle se formule à travers le double objet dont rend compte le terme "discipline » : l'organisation à laquelle on doit se conformer pour participer au monde des savoirs, et le rapport pédagogique du maître à l'élève-discipulus (Fabiani, 2006). C'est que dans le monde scientifique l'innovation n'est pas le quotidien de l'activité. Elle repose sur des activités de reproduction aussi bien que d'extension des savoirs. 


\section{Dans les pratiques historiennes}

Dans le champ des historiens, des études historiques montrent que le rapport entre sujets à propos du rapport entre sujet et objet se fonde sur le développement de systèmes sémiotiques qui organisent la communauté. Un exemple paradigmatique est celui de l'étude de Grafton (1998) qui expose comment au fil des siècles le système de la note en bas de page symbolise la modification radicale de l'organisation des échanges et, simultanément, les critères de validation du savoir sur le passé. Le dialogisme que soustend cette modalité de travail du passé s'organise dans l'articulation des sources primaires et des récits explicatifs de l'historien (objet/sujet) par le truchement d'un dialogue critique avec les interprétations existantes ; articulation matérialisée selon des modalités variables dans l'espace de la page et du texte, mais toujours présente sous la forme d'un double récit : de l'objet et de l'enquête sur l'objet.

De la même manière, l'enchâssement de la forme séminaire développée à partir du $\mathrm{XIX}^{\mathrm{e}}$ siècle par l'historien allemand Ranke, dans l'organisation de la communauté disciplinaire rend compte d'un système spécifique d'articulation du social et de l'épistémologique. Comme le montre l'étude de Risbjerg Eskildsen (2007), le séminaire devient le lieu qui institue une certaine stabilité à la tension tradition/ innovation en obligeant les novices à reproduire les activités du maître, tout en participant à la relation du groupe au reste de la communauté historienne (passée et présente) dans un rapport de différenciation et donc d'innovation.

Ces deux études historiques de la discipline donnent corps à la double dimension sociale et cognitive des principes pratiques qui fondent actuellement l'histoire savante, et que formule de manière synthétique Galvez-Béhar : "L'histoire est une connaissance des sources et non du passé [...] Les sources ou les documents sont à considérer dans toute leur variété et l'on ne saurait les limiter à un seul type. En outre, ils ne s'offrent jamais spontanément à notre connaissance : ils ne sont appréhendés que dans le cadre d'une démarche heuristique où la théorie et le questionnement sont fondamentaux. Aussi, la connaissance de la source est-elle d'abord celle des conditions qui rendent possible l'appréhension d'un document par l'historien » (2009, p. 110-111).

\section{Dans les pratiques scolaires}

Ces éléments permettent finalement de revenir aux pratiques scolaires afin d'envisager la mise en relation des principes pratiques de classe et des principes structurants de référence dans notre problématique de l'articulation du social et du cognitif.

Les principes pratiques de la classe d'histoire tels que ceux envisagés précédemment sont des modèles générateurs «sur le papier »-pour le didacticien qui rendent compte du sens du jeu « faire de l'histoire en classe », celui qui génère les actions des acteurs de la classe. Dans le cadre bourdieusien des pratiques sociales, ces principes pratiques rendent possible l'appréhension des pratiques ordinaires de la classe d'histoire comme reproduction ${ }^{14}$ : mise en œuvre d'une tradition disciplinaire. C'est en ce sens que ces seuls principes pratiques étudiés, dans le cadre de la théorie de la discipline scolaire, ne permettent pas d'envisager la description et la compréhension (des conditions) de sorties des pratiques ordinaires, comme par exemple les moments de problématisation dans la classe. Cette limite à la perspective sociale que porte la notion de discipline scolaire, même vue à travers les principes pratiques comme nous l'avons proposé au début de ce texte, est le symétrique de la limite constatée de la perspective portée par les espaces de contraintes dans la logique des savoirs. Le déterminisme social est le pendant du déterminisme épistémologique.

C'est face à ce double blocage de la dualité social/ cognitif que l'analyse en termes de champ peut ouvrir des pistes : la spécificité de la pratique théorique repose, on l'a vu, sur le fait que les principes pratiques sont eux-mêmes un enjeu des pratiques. On peut donc, sur cette base, renouveler notre hypothèse des principes pratiques des élèves comme obstacles à l'appropriation des principes structurants de la discipline. Toute problématisation scientifique suppose une rupture avec les idées initiales, mais cette rupture doit être un enjeu explicite dans le travail scientifique. En conséquence, l'émergence des principes pratiques des élèves devient une condition à une activité de problématisation. À cette première condition s'adjoint une seconde : la mise en question de ces principes pratiques doit se faire en faveur des principes structurants de référence.

L'exploration de cette problématique n'a de sens qu'à travers l'étude d'un cas (Bourdieu, 1992, p. 249-259). Celui que nous proposons maintenant 
est élaboré à partir d'un corpus de données, issu d'une situation forcée construite dans le cadre théorique de la problématisation. Sa construction repose bien entendu sur les éléments problématiques que nous venons d'exposer.

\section{La Première guerre mondiale à l'école élémentaire : une séquence forcée pour faire problématiser}

Selon les principes des situations forcées (Orange, 2010, p. 77), la séquence est définie par l'enseignante et l'équipe de chercheurs dans ses visées d'apprentissage et dans les objectifs de recherche du groupe. Le programme du cycle 3 de 2008 fait de la violence le concept explicatif qui unit les deux guerres mondiales au $\mathrm{XX}^{\mathrm{e}}$ siècle, ce qui justifie qu'on s'interroge sur ce qui fait de la Grande Guerre un événement marquant pour les contemporains ${ }^{15}$. La séquence préparée introduit une démarche d'étude de cas susceptible de favoriser une démarche critique à l'égard de l'explication dominante dans l'enseignement de la guerre de 14-18 (guerre totale, nombre de morts, expérience dramatique de la guerre par les Poilus considérés comme les premières victimes). Faire comprendre différemment l'expérience de guerre vise à installer les élèves dans un paradigme intégrant la question de la violence - subie et exercée - tel qu'il est développé par une histoire culturelle et sociale. Il s'agissait donc d'amener les élèves vers l'explication des historiens dans une démarche d'apprentissage par problème, et de leur faire produire un texte de savoir porteur de ce problème historique. Le corpus est constitué des documents de la séquence, des productions des élèves, des enregistrements vidéo de deux classes (A et B) et de leurs transcriptions.

Le groupe a ajusté les différentes situations au fur et à mesure, et la séquence s'est organisée de la façon suivante :

\begin{tabular}{|c|c|}
\hline déroulement & corpus \\
\hline $\begin{array}{l}\text { Séance } 1 \\
\text { Pourquoi l'appelle-t-on la Grande } \\
\text { Guerre? Hypothèses discutées collec- } \\
\text { tivement avant d'enquêter sur quelques } \\
\text { images que disent-elles des hypothèses? } \\
\text { Dossier « René Jacob» à lire pour la S2. }\end{array}$ & $\begin{array}{l}\text { Notes sur les } \\
\text { cahiers photo- } \\
\text { copiés (travail } \\
\text { individuel, puis } \\
\text { après discussion } \\
\text { collective) } \\
\text { Enregistrement } \\
\text { audio et vidéo et } \\
\text { transcription } \\
\text { Tableau filmé } \\
\text { Documents du } \\
\text { dossier }\end{array}$ \\
\hline $\begin{array}{l}\text { Séance } 2 \\
\text { L'expérience de guerre de RJ } \\
\text { construire des catégories collectives pour } \\
\text { en parler (vu, fait, ressenti, compris, } \\
\text { exprimé) } \\
\text { Consigne } \\
\text { en groupe, que peut-on dire de l'expé- } \\
\text { rience de guerre de RJ à partir de ces } \\
\text { documents? La notion d'expérience est } \\
\text { discutée et déclinée en quelques catégo- } \\
\text { ries avant le travail de groupe qui vise à } \\
\text { produire des affiches donnant du contenu } \\
\text { à ces catégories (colonne de droite) et } \\
\text { justifiant par référence au document } \\
\text { utilisé (colonne de gauche). } \\
\text { Affiches présentées et discutées pour } \\
\text { ranger les éléments d'informations par } \\
\text { catégorie (au tableau par la maîtresse avec } \\
\text { des couleurs). }\end{array}$ & $\begin{array}{l}\text { Enregistrement } \\
\text { audio et vidéo et } \\
\text { transcription } \\
\text { Affiches } \\
\text { produites } \\
\text { en groupes } \\
\text { (tableaux) }\end{array}$ \\
\hline $\begin{array}{l}\text { Séance } 3 \\
\text { Qu'est-ce qui permet de généraliser } \\
\text { cette expérience aux autres Français de } \\
\text { l'époque? Faire des hypothèses } \\
\text { Un tableau synthétise ce qui a été fait } \\
\text { en S2 et est distribué aux élèves. On } \\
\text { y retrouve les catégories (colonne de } \\
\text { droite). Colonne de gauche, question- } \\
\text { titre pour généraliser ils doivent faire des } \\
\text { hypothèses. } \\
\text { En fait, dans les deux classes, la consigne } \\
\text { se transforme à partir de l'exemple } \\
\text { produit en début de séance : il s'agit fina- } \\
\text { lement de différencier les situations des } \\
\text { soldats du point de vue civil et militaire } \\
\text { (en première ligne ou artilleur, célibataire } \\
\text { ou père de famille, etc.). C'est ainsi que } \\
\text { sont remplis les tableaux. }\end{array}$ & $\begin{array}{l}\text { Enregistrement } \\
\text { audio et vidéo et } \\
\text { transcription } \\
\text { Tableaux } \\
\text { complétés }\end{array}$ \\
\hline
\end{tabular}

Notre analyse a posteriori repose sur la mise en œuvre de notre catégorisation entre principes pratiques des élèves et principes pratiques des historiens en référence aux principes structurants. À cet égard, elle s'appuie sur la reformulation de l'analyse a priori des enjeux historiques. Puis l'analyse côté élèves doit nous permettre de mieux caractériser les spécificités 
de la problématisation historienne scolaire et les conditions de son appréhension. Elle doit également nous permettre de valider l'hypothèse du double obstacle épistémique et social que constituent des principes pratiques dans la classe.

\section{La question historiographique : analyse a priori}

La compréhension des enjeux liés aux débats historiens ayant porté sur l'objet Grande Guerre dans les années 2000 est nécessaire pour saisir comment ils se trouvent réifiés dans l'espace scolaire sous la forme de deux thèses contrainte vs consentement qui sous-tendent le renouvellement proposé dans les programmes (Le Marec \& Vézier, 2006). Cette réification masque en réalité l'évolution des principes.

Derrière le choix d'inclure dans l'objet particulier de la Grande Guerre une dimension anthropologique et de faire l'histoire de la violence, se joue la nature de l'explication historique liée au changement de paradigme. Le recentrage sur une histoire prenant en compte les acteurs, sans les réduire au statut simplificateur de victimes, donne lieu à une controverse sur la réponse à apporter à la question induite par ce changement historiographique : comment les soldats ont-ils tenu dans les conditions de la vie combattante (Prost \& Winter, 2004 ; Prost, 2006) ? La question naît dans les années 1990, à partir d'un constat d'ordre anthropologique : avec la disparition des derniers témoins, l'expérience que fut cette guerre devient complètement étrangère aux générations qui ne l'ont pas connue directement. De ce fait, une " rupture cognitive " nous rend inaccessible le système de représentations des contemporains, alors que le retour de la Grande Guerre dans la conscience collective française implique une proximité avec les victimes, Poilus et mutins (Audouin-Rouzeau \& Becker, 2000, p. 18-19). Ce qui pose problème se situe ici au niveau des principes qui permettent d'appréhender le passé révolu : l'historien doit penser la distanciation, l'estrangement (Ginzburg, 2001), la rupture, là où la pensée sociale commune - et la mémoire - conçoit continuité et proximité. Pour sortir de ce paradoxe, un double effort est tenté pour "faire de l'histoire ": se doter des outils conceptuels qui manquent pour comprendre le fait guerrier et expliquer la violence de masse, et, de façon complémentaire, changer le rapport épistémologique qui lie les témoignages et l'interprétation. Les débats entre historiens sur l'objet étudié sont suscités par une approche très critique envers une historiographie née de "la dictature des témoignages », fondée sur une centration sur les soldats et leurs points de vue, aboutissant alors à majorer la figure victimaire du Poilu ${ }^{16}$. Dans la récusation d'une historiographie par une autre, se joue la remise en cause du principe structuraliste, où les agents historiques ont peu de poids, par une histoire attentive aux marges de liberté des acteurs.

Se détournant autant d'un contexte déterminant et exclusif que de comportements renvoyant à des choix individuels, l'historien cherche à dégager les rationalités des acteurs historiques. L'accent est mis sur l'engagement des sociétés dans le conflit et le consentement à la guerre. C'est en contestant cette focalisation sur l'engagement des individus pour leur nation que la thèse inverse de soldats tenant par la contrainte s'est développée. Cette divergence d'interprétations fait alors de la mise en tension entre sources et récit un enjeu central (Buton, Loez, Mariot, \& Olivera, 2008 ; Audouin-Rouzeau, 2009). L'appartenance au même cadre explicatif, l'histoire culturelle attentive aux pratiques signifiantes - « elle étudie comment les hommes et les femmes ont conféré un sens au monde dans lequel ils vivaient " (Prost \& Winter, 2004, p. 47) - réduit la portée de l'opposition entre deux principes : l'explication par le contexte historique et l'ancrage dans le réel, opposée à l'explication par une structure à travers le concept de culture de guerre $^{17}$. Ce qui est critiqué dans ce concept, c'est la visée d'objectivation et une position surplombante de l'historien qui ne permet pas de prendre en compte les discours particuliers, les choses non dites, les pratiques, tout ce qui donne accès aux raisons de l'autre $^{18}$. Or, ce risque de surplomb s'accompagne d'une coupure entre le système de représentations et les pratiques, ce qui est sans doute aggravé dans la réification de la culture de guerre transposée dans les programmes du secondaire.

Il n'est donc pas inintéressant pour la compréhension de ce qui se travaille dans la classe de resituer l'évolution des principes, en cherchant à l'exprimer avec les outils de la problématisation. Considérons l'explication classiquement donnée au problème historique évoqué plus haut. Parmi les données, on souligne l'importance du nombre global de morts et de blessés (cf. les bilans et les tableaux classiquement présents dans l'enseignement de la question). Plus la guerre dure, plus le conflit se durcit par un 
long processus de totalisation (plus de ressources, plus d'armes meurtrières). Ce modèle explicatif du nombre de victimes est tout à fait compatible avec le découpage entre guerre de mouvement, suivie du moment identifié comme " guerre des tranchées ", puis reprise de la guerre de mouvement. Cela renvoie à la mise en relation communément faite entre une durée et l'accentuation (ou le déclin) d'un phénomène. Prost complète l'explication par l'apprentissage du métier de soldat par tous ces civils mobilisés ou volontaires. La figure du Poilu agrège tout cela, en masquant les différences entre soldats au profit de la différence de l'expérience du Front avec celle de l'arrière. L'explication par le principe du déterminisme de la situation l'emporte dans ces raisonnements et laisse peu de place aux choix des acteurs ${ }^{19}$.

La rupture opérée avec cette thèse s'est faite sur le plan épistémologique en discutant la validité des témoignages, mais de façon plus générale, elle engage un changement de principe méthodologique structurant au sens de Orange-Ravachol et Beorchia. En effet, l'« étrangeté » revendiquée par l'historien, réintroduit de la distance et oblige à modifier tant les données que les modèles explicatifs. C'est ainsi que des bilans plus précis des victimes, mois par mois, année par année, sont établis. La mise en évidence d'un plus grand nombre de morts en 1914 rend caduque la thèse précédente. Un autre modèle explicatif est proposé, celui du franchissement de seuils de violence à différents moments de la guerre (invasions, retraites). Contrairement au processus de civilisation rendant difficilement compréhensible le rapide passage à la violence de guerre, il y a eu un basculement rapide dans la violence qu'il s'agit alors d'expliquer. Ces allers-retours entre données et modèles conduisent à changer de mode de périodisation et à ne plus voir dans le seul contexte, sous la forme de la durée de la guerre, la cause de la violence. La théorisation d'une culture de guerre intériorisée amène à chercher de nouveaux objets, de nouvelles sources comme les jouets ou les objets, et cette culture n'est pas vue comme une conséquence d'un processus de totalisation mais comme l'origine de la violence. C'est un retournement par rapport à la vision des mentalités venant en dernier dans les démarches d'histoire sociale.

La vivacité des débats entre 2000 et 2008 s'explique par la résistance du principe de la détermination par la situation - ils n'avaient pas le choix face à l'idée d'une culture de guerre intériorisée - ils consentent - intégrant des éléments antérieurs à l'éclatement de la guerre comme le patriotisme et la haine de l'autre. Un tel principe d'attention aux acteurs oblige donc à modifier les temporalités dans lesquelles on étudie la guerre, et il s'affranchit en partie de la situation. Si déterminisme il y a, il est d'une autre nature.

La sortie d'un débat se fait par une " négociation de la distance entre les hommes à propos d'une question, d'un problème », façon dont le philosophe M. Meyer définit la rhétorique (Fabre, 2006). Pour l'appréhender, Fabre, à la suite de Meyer, propose de dépasser le plan des solutions et même celui de l'argumentation pour remonter au problématique. Ainsi dans la question historique, discuter de consentement ou de contrainte serait en rester au niveau des réponses à la question « comment ont-ils tenu ? », sans remonter au problématique. Ce qui s'explique, selon l'analyse de Prost (2006), par le fait que les tenants de chaque interprétation se situent en réalité au sein d'une même configuration historiographique ${ }^{20}$. Pour Prost, il est plus fondamental pour la compréhension des changements de l'objet Grande Guerre, de saisir en quoi cette histoire culturelle intéressée aux individus et à leur vécu s'oppose à la $1^{\text {re }}$ configuration, celle d'une histoire militaire et nationale, non seulement en réinterprétant les intentions des acteurs à partir des représentations qui les sous-tendent, mais au fond en posant la guerre à une échelle autre que le national (guerre civile européenne, combattants européens). La construction du problème est fondamentalement différente dans le choix des données ${ }^{21}$ et ne se limite pas au passage d'une recherche des causes et des responsabilités à une compréhension des raisons d'agir des acteurs. Les raisons avancées ne prennent ainsi sens qu'à l'intérieur du registre explicatif propre à chaque configuration identifiée. À des fins de contrôle du récit s'impose alors à l'historien la « nécessité d'un va-et-vient permanent entre la simplification théorique et la prise en compte de situations et contextes particuliers » (Revel, 2009). C'est donc dans le cadre d'une historiographie attentive à l'action et aux acteurs qu'on repère un principe pratique essentiel aux approches actuelles de la guerre : l'historien travaille en effet le rapport entre actions et contextes; autrement dit, il ne cherche pas les causes dans des contextes déterminants ou dans des choix individuels, mais il tente d'appréhender les ressorts du passé en décrivant les situations comme 
des rencontres singulières entre actions et contextes dans lesquels ces acteurs s'inscrivent (Vézier, 2012, Delacroix, 2010). La notion de principe pratique permet ainsi d'identifier le problématique comme nous avons essayé de le faire plus haut.

Le dépassement de l'alternative contrainte/ consentement se fait différemment chez les élèves, car il leur est moins aisé de poser l'histoire de la guerre de 1914-1918 comme un objet à questionner, comme il leur est difficile de sortir du déterminisme de la situation.

Les récits produits dans la classe en décalage avec le texte des historiens

La problématisation est avant tout un processus mais il nous faut regarder d'abord le savoir qui se construit dans la classe et pour cela prendre en compte les productions textuelles comme traces de l'activité de réorganisation du savoir par les élèves à la séance 4. Le texte problématisé se veut un ensemble d'énoncés vrais, qui prennent sens en donnant les raisons, et par-là le texte est porteur d'une généralisation qui le détache des conditions locales dans lesquelles il a été élaboré. Pourtant la textualité dominante en classe, en privilégiant des savoirs propositionnels, masque les pratiques qui l'ont fait naître, de même que les contraintes textuelles, notamment l'organisation du texte, imposent de ne retenir que les faits qui peuvent s'y plier (Rey, 2002). Cependant dans notre cadre, pour problématiser il faut que les textes gardent une trace des pratiques pour que la réponse travaillée puis validée dans la classe ne se coupe pas du problème. Réfléchir didactiquement aux conditions de possibilité de tels textes nécessite de penser l'articulation entre l'enquête et le récit historique ${ }^{22}$. Dans cette pensée de l'articulation de la pratique au texte, il convient de ne pas oublier que le texte renvoie aussi à des pratiques différentes de celles de la classe. Ainsi Rey s'intéressant à la transformation d'un savoir, en l'occurrence un concept, montre le rôle décisif de la mise en texte de ce savoir : " c'est le fonctionnement textuel qui permet que des concepts, ainsi isolés de la pratique qui les a vus naître, puissent recouvrir une pratique radicalement différente $»($ Rey, 2002, p. 43).

La caractéristique anthropologique du savoir à être mis en texte ne doit pas masquer que les textes peuvent se distinguer selon leur degré de généralité (ou de théorie) mais également selon le degré plus ou moins étroit avec le problème, et les traces des pratiques. Les textes des élèves demandés à la fin de la séquence pourraient ainsi relever davantage du type rapport de recherche que du texte théorique ou du texte de vulgarisation. La question est de savoir si ces textes peuvent nous dire quelque chose du degré de problématisation auquel les élèves ont individuellement accédé, ou s'il y a échec final de la problématisation, avec de simples savoirs propositionnels.

\section{La mise en cohérence textuelle et l'empirisme l'emportent dans un texte de savoir peu problématisé}

Traiter de la violence de la guerre de 14-18 dans la perspective du $\mathrm{XX}^{\mathrm{e}}$ siècle implique de se centrer sur l'expérience combattante dans sa diversité, et s'oppose à la tradition scolaire par laquelle on aborde les Poilus et ce qu'ils ont souffert en tant que masse combattante. Pour les élèves, cela se traduit par une conception dominante de la situation de guerre qui fait des soldats des victimes : ils ont peur, ils souffrent dans les tranchées, ils perdent leurs camarades, ils sont blessés ou tués. Passer d'une configuration centrée sur la guerre totale à la configuration marquée par l'anthropologie historique, nécessite de resituer cette expérience dans une expérience globale où le soldat est aussi celui qui tue. C'est dans cet objectif que nous avons introduit l'étude de cas d'un soldat ayant existé, nommé René Jacob, pour amener les élèves à remettre en cause leur vision des Poilus. Problématiser supposait alors s'appuyer sur le savoir déjà là pour l'expliciter et le constituer en obstacle, contraignant à construire le problème historique autrement. Pour les élèves, les réponses fournies à la question de la première séance - qu'est-ce que les soldats ont vécu de la guerre pour l'appeler la Grande Guerre ? - sont renforcées avec l'étude (séance 2) de l'exemple du soldat qui ne parvient pas à constituer un cas critique questionnant leur conception victimaire.

Nous y voyons un effet de la consigne qui consiste à prélever des informations dans les documents. Après avoir éclairci avec les élèves ce qu'on peut entendre par " expérience de guerre », l'enseignante leur demande de « réaliser des affiches pour essayer de donner tous les éléments de l'expérience de guerre de René Jacob [... ] il ne s'agit pas de rajouter d'autres 
choses, c'est vraiment ce qu'on voit dans les documents ». Ils doivent, leur dit-elle, "pour chaque élément noter ce qui dans les documents ou dans tel document, $[\ldots]$ vous a permis de dire ça. Donc ça veut dire qu'il faut se mettre d'accord là-dessus ». Ce travail effectué sur le dossier de sources primaires aboutit à caractériser l'expérience de guerre mais se trouve transformé en simple texte ${ }^{23}$. Cela correspond à un rapport habituel aux documents dans lesquels les élèves puisent des éléments concrets de ce qu'ils pensent être le réel du soldat, au détriment de tout rapport distancié à la source, susceptible d'élever un désaccord au sein des groupes. L'effacement de la médiation des traces réduit alors la distance entre le passé des soldats et le présent de la classe, et par ailleurs transforme René Jacob en simple exemple. Une simple mise en cohérence textuelle se substitue au travail d'enquête à effectuer. Le travail de sélection puis de catégorisation de l'expérience, mené collectivement, dans cette deuxième séance, va donc dans le sens des principes pratiques identifiés dans les pratiques ordinaires.

La séance 3 est initialement construite pour faire rupture en prenant appui sur « ce qu'on sait maintenant » à l'issue du travail de catégorisation. En posant la question des conditions de la généralisation, il s'agit d'inciter la classe à revenir sur l'usage des documents qu'elle a mis en œuvre dans la séance 2. Autrement dit, il s'agit de faire travailler les conditions d'appréhension des documents pour parler de la violence de la Première guerre, et pas seulement pour René Jacob. En particulier, on visait un approfondissement de la prise en compte de la lettre à sa famille non comme un simple témoignage, mais un échange épistolaire et de ce fait, la mise en évidence de savoirs sur les relations entre front et arrière (par exemple sur le fait de taire tout ce qui a trait à l'action de tuer). L'enseignante dégage en début de séance 3 qu' « il y a une idée qui ressort vraiment, c'est la violence, et la violence pour le soldat, mais pas seulement, la violence pour un peu tout le monde en fait ». Les élèves doivent faire des hypothèses pour dire en quoi leurs explications pour le soldat seraient généralisables. La transformation des éléments de la réalité individuelle et collective en faits historiques nécessite de pouvoir généraliser cette expérience de violence vécue, subie, voire commise, en se confrontant au modèle de victime et aux données. La démarche vise donc à faire émerger les conditions de validité d'une généralisation et à construire une explication d'ordre historique.
Ce faisant, les élèves sont confrontés à un problème nouveau correspondant à la logique des savoirs en jeu et également à un changement de situation didactique. Entre les séances 1 et 2, dans le dénivellement produit par le passage d'une réalité donnée comme globale et vraie (ils sont nombreux à mourir et à être blessés) à la singularité de l'expérience, puis à la séance 3 , dans le questionnement de la généralisation par rapport au cas, les élèves se trouvent face à l'incertitude des réactions des agents historiques confrontés à la violence en guerre. La réponse n'est plus si évidente. Comprendre l'expérience de guerre du Poilu, c'est comprendre qu'il est un civil confronté à la guerre, ce qui passe par la saisie d'une réalité qui dépasse le document et donc par la rupture avec la validation directe par l'information tirée du document $\left(\mathrm{S} 2 \mathrm{~A}^{24}\right)$ :

\begin{tabular}{|l|l|l|}
\hline 143 & M & $\begin{array}{l}\text { Tu veux dire sinon ce serait pas marqué } \\
\text { dans le document qu'on a. Je t'arrête } \\
\text { tout de suite, ces documents n'ont pas } \\
\text { été faits pour que les élèves trouvent } \\
\text { des choses. Ce sont des documents réels } \\
\text { et c'est de ces documents là qu'on va } \\
\text { essayer de comprendre des choses. C'est } \\
\text { à nous de décider si on pense que c'est } \\
\text { quelque chose d'important pour son } \\
\text { expérience ou pas. [...] }\end{array}$ \\
\hline
\end{tabular}

Du point de vue de l'enseignante, cela engage clairement une activité différente de la prise d'information, mais sans donner aux élèves les moyens de se repérer dans ce nouveau travail. Une discordance apparaît de fait au cours du récit qui se construit dans la classe, alors que l'enseignante vise à faire de l'articulation de la signification et des données un enjeu. On voit pourtant que cette situation interpelle certains élèves, parce qu'elle vient aussi perturber ce qui est une règle dans les activités de la classe, comme le manifestent ces élèves entendus en arrière-plan et ce qui autojustifie le travail : en classe d'histoire, on travaille avec des documents, c'est même ce qui caractérise la discipline aux yeux des élèves, et on utilise des exemples, mais sans que les élèves se questionnent ordinairement sur la valeur de ces exemples et de ces documents. De ces derniers on infère ordinairement les faits du passé. Et on a l'habitude de travailler sur les « grands hommes » $(\mathrm{S} 2 \mathrm{~A})$ :

\begin{tabular}{|l|l|l|}
\hline 213 & Zoé & $\begin{array}{l}\text { Mais madame, pourquoi vous nous } \\
\text { avez, qu'est-ce qu'il a de spécial RJ } \\
\text { pour que vous nous fassiez travailler? } \\
\text { C'est juste un soldat comme les autres } \\
\text { ou c'est quelqu'un d'exceptionnel ? }\end{array}$ \\
\hline
\end{tabular}




\begin{tabular}{|l|l|l|}
\hline 218 & Zoé & $\begin{array}{l}\text { Oui c'est un peu comme lui, parce } \\
\text { que en général on étudie les plus } \\
\text { grands, [d'autres élèves interviennent: } \\
\text { les rois, César], ce serait bien de se } \\
\text { baser un peu sur les autres, les plus } \\
\text { bas, comme ça on verrait vraiment } \\
\text { ce qu'ils ressentent ceux qui sont en } \\
\text { dessous. }\end{array}$ \\
\hline 219 & M & $\begin{array}{l}\text { C'est très intéressant, on finit } \\
\text { là-dessus. }\end{array}$ \\
\hline
\end{tabular}

Ce type d'échange ne suffit pas à réorienter la dynamique du récit en faisant de l'exemple un cas questionnant l'interprétation des Poilus comme victimes. L'étude du dossier de René Jacob leur fournit l'intérêt de parler aussi des anonymes pour rééquilibrer la place faite aux héros ou aux grands hommes comme ce fut le cas dans l'historiographie scientifique et scolaire jusqu'au milieu du $\mathrm{XX}^{\mathrm{e}}$ siècle et au-delà. Cette préoccupation renvoie d'ailleurs aux pratiques sociales aux lendemains de la guerre qui font des soldats les héros de 14-18. Les élèves argumentent sur le plan social, sans qu'il y ait réflexion épistémologique. Ils intègrent dans le récit le point de vue de soldats de base parce que c'est plus vivant et plus complet (218). Se poser la question de l'exemplarité de René Jacob (213) ne débouche pas sur un nouveau problème, tout au plus cette question introduit-elle la tâche de généraliser l'expérience du soldat. Durant cette séance 3, le choix didactique peu ordinaire de faire travailler des élèves sur les conditions de la généralisation se trouve modifié dans le processus de construction par l'enseignante. En réalité, celle-ci élude très rapidement la question directe des conditions à la généralisation et conduit ses élèves à explorer une multiplicité d'exemples, aux côtés de René Jacob, comme si on s'appuyait sur plusieurs soldats (celui qui est célibataire, celui dont la famille est en zone occupée, celui qui est au front, celui qui est artilleur, celui qui s'occupe des blessés et des morts, etc.). Dès le début de la séance, elle prend collectivement un exemple issu du travail de la séance 2 (« ce qu'il a vu») pour questionner, hors documents, ce que « les autres Français » auraient pu voir (S3A) :

\begin{tabular}{|l|l|l|}
\hline 5 & M & $\begin{array}{l}\text {.. Ce que je vous propose pour démar- } \\
\text { rer pour que tout le monde comprenne } \\
\text { bien comment il faudra faire le travail, } \\
\text { c'est qu'on regarde ensemble la première } \\
\text { chose, dans ce qu'il a vu. Première } \\
\text { chose, Marc. }\end{array}$ \\
\hline 6 & Marc & Des cadavres. \\
\hline
\end{tabular}

\begin{tabular}{|l|l|l|}
\hline 7 & M & $\begin{array}{l}\text { Des cadavres, on s'arrête là, alors il a vu } \\
\text { des cadavres, ça c'est ce qu'on a pu tirer } \\
\text { des documents. Il a vu des cadavres. } \\
\text { Qu'est-ce qui permet de généraliser ? } \\
\text { Est-ce qu'on peut généraliser ça, le } \\
\text { fait d'avoir vu des cadavres aux autres } \\
\text { Français de l'époque? }\end{array}$ \\
\hline
\end{tabular}

Ainsi retraduite par l'enseignante et comprise par les élèves, la consigne est, comme dans la séance 2 , faiblement orientée vers le processus de problématisation. Dans la suite de la séance, les élèves accomplissent cette tâche de description d'autres cas possibles sans qu'émergent des questions liées aux documents. Or ce qui fait avancer le savoir, ce sont moins les tâches que les questions. Et en histoire, ce sont les questions qui amènent à reconsidérer les sources potentielles et à reprendre certaines données autrement, en les confrontant aux interprétations précédentes dans la logique dialogique évoquée plus haut. Au contraire dans le corpus, la classe reste dans le registre empirique, alors que l'interprétation de l'empirie imposerait le recours au registre explicatif car c'est là que se donnent les choix de l'interprétation. De plus, en l'absence des contrôles critiques, les raisons construites ne sont pas historiques mais seulement en cohérence entre elles et avec le savoir qui circule dans la classe. Sous cet angle les textes rédigés à la séance 4 relèvent d'une cohérence textuelle élargie aux lectures de certains élèves. Ils montrent que la visée de généralisation à la séance 3 n'a pas provoqué de changement de problème pour les élèves car la figuration comme victime n'est pas vraiment remaniée par l'introduction du soldat qui tue, à côté du soldat tué, blessé, souffrant.

\section{Des textes qui ne remettent pas en cause l'interprétation dominante}

Prendre en compte des textes produits c'est, dans notre analyse, chercher à dépasser le constat habituel de l'écart entre mise en texte dans la classe et textes des élèves. Ces textes viennent plutôt confirmer que la problématisation attendue de la démarche de l'étude de cas n'a pas pleinement fonctionné. Du savoir a bien été produit mais il n'est pas entièrement historique, avons-nous souligné plus haut. Ces textes nous intéressent en ce qu'ils font trace de la façon dont chaque élève réorganise plus ou moins des éléments d'origines diverses, et rendent visible - y compris en creux - l'impact des principes pratiques 
dans la compréhension du fait historique. Dans notre démarche d'analyse, un aller-retour est nécessaire entre les séances 2, 3 et 4, car l'analyse de ces textes ne peut se réduire ni à une analyse textuelle, ni à les considérer comme simple produit dans une logique linéaire. Ce que nous dégageons dans un premier temps de ces textes, nous le replacerons donc ensuite dans une perspective plus globale, tenant compte de notre questionnement sur les principes.

La figure réductrice du Poilu est un élément indispensable dans la plupart des récits, mais elle est en soi faiblement explicative des contradictions de l'expérience de guerre. Les textes produits relèvent en effet, comme à la séance 2 , d'une conception de l'histoire où l'on retrouverait la réalité vraie du passé, sans la discuter. D'où vient alors la diversité des solutions manifestées par les écrits des élèves ayant répondu à la question "Que savons-nous maintenant des soldats au combat? » (onze textes dans la classe A) ?

Il est frappant de lire les intrigues qui soustendent les textes à partir des éléments choisis et ceux écartés de fait. Six textes mettent en avant l'expérience de guerre difficile ( « beaucoup sont morts », " c'est dur », « ils vivaient des choses affreuses »), les cinq autres commencent par la réalité militaire (front, arrière, «ils lançaient des grenades »). En cherchant à intégrer l'expérience de guerre dans la chronique militaire, ils affirment ainsi que le soldat a peu de poids sur le déroulement des hostilités. Très peu parviennent à catégoriser des acteurs si ce n'est par juxtaposition, sans doute en lien avec le traitement de la séance 3 évoqué plus haut. Lorsque les élèves restituent la figure de soldat-victime, ils le font en intégrant des éléments qui proviennent du travail collectif mais aussi de leurs lectures. À côté de la lettre de René Jacob, le roman Un tirailleur en enfer alimente cette vision. Certains élèves comme Noémie ont retenu l'argument qu'être des civils, des non professionnels de la guerre, avec une famille, est un facteur aggravant la peur.

Plus rares sont les écrits qui reprennent à leur compte le paradoxe créé par le fait d'être un civil et évoquent l'autre figure possible, des soldats qui tuent. Dans le texte de Zoé, argumenté et témoignant d'un effort de raisonnement, la guerre transforme les civils, " donc pas habitués à tuer », en soldats capables de « commettre des actes horribles». Le reste du texte est un tissage d'éléments venus des documents, du travail collectif et de la lecture du roman, dans la logique des séances 2 et 3 qui ont fonctionné vérita- blement comme une machine à intégrer les données en balayant des contextes possibles en situation de guerre. Ces éléments ont fonctionné comme des « savoir que » et non pas des « savoir pourquoi cela n'a pas été autrement $»$. Il semble logique que le texte final montre la résistance de l'interprétation dominante. En effet, les deux figures contradictoires s'additionnent sous l'effet du principe déterministe : les soldats « obligés » de tuer sont aussi des victimes de la situation de guerre, sans discuter l'éventuelle contradiction, et en puisant dans des explications de nature empirique.

«Beaucoup sont morts au front : et pas seulement des Français : aussi les soldats des colonies. Ils étaient obligés de commettre des actes horribles et la plupart des soldats n'en étaient pas ; ils étaient des civils, avant. Donc ils n'étaient pas habitués à tuer, et pas non plus à courir sous des pluies de balles. Ils voyaient leurs amis, leurs compagnons d'armes, tomber pour ne plus se relever, dans cette terre mutilée et arrosée de sang. La vie était un enfer dans les tranchées, et certains se mutilaient ou se mutinaient [...]». (Zoé)

De son côté, Tanguy pose clairement l'alternative, tuer ou être tué, mais en fait lui aussi un effet de la contrainte liée à la situation - « ils tuent les ennemis et les soldats recrutés comme René Jacob ont du mal à se faire de tuer pour survivre »- et se réfère à une explication possible du comportement de survie - « certains disent 'on est comme des fauves, tués ou être tués $»$. Il intègre donc la réticence potentielle des soldats, il les montre face à des choix d'action (fuir, se mutiler). Faisant des soldats des agents possibles de l'action, il emploie le présent qui contraste avec l'imparfait de ses phrases plus descriptives de la guerre. S'il témoigne d'un raisonnement logique (alors que, mais), l'organisation générale ne rend pourtant pas compte des raisons de renoncer au modèle de la victime. Nicolas et Sophie, quant à eux, identifient l'autre contradiction entre le fait d'écrire aux familles et le fait de ne pas tout dire, entre la contrainte hiérarchique (censure) et le mensonge du soldat, mais sans réussir tout à fait à l'expliquer.

L'enrichissement des connaissances est patent, des explications sont fournies, mais sous une forme propositionnelle et en restant au registre empirique. La modélisation reste ainsi implicite et secondaire, parce qu'au fond c'est en se mettant à la place des soldats que les élèves répondent à la question. Ainsi, 
« pour moi, la guerre, c'est l'enfer » conclue Zoé. Les élèves ont effectivement sélectionné ce qui leur paraissait pertinent, réorganisant leur texte en continuité avec leur expérience personnelle, et sans se soucier de contrôler ces importations. La métaphore de l'enfer n'est pas un concept historique, on peut donc faire l'hypothèse qu'elle vient faire obstacle à ce principe de tâtonnement conceptuel évoqué dans les travaux de Lautier. Elle n'a pas été travaillée, bien qu'elle porte un récit (celui de certains témoins, relayé par le roman historique) qui aurait pu être mis en contradiction avec le récit porté par les savoirs validés dans la classe.

Mais il est difficile pour les élèves de rédiger un texte problématisé qui se confondrait avec un véritable récit historique car celui-ci suppose une autonomisation de l'explication et une dissociation avec le temps de l'action (Ricœur, 1983, p. 318). Ce qui fait office de principe structurant dans l'enquête historienne, la distanciation, ne peut donc pas être évacué dans la mise en texte finale, qui doit être aussi suffisamment générale pour ne plus dépendre du travail fait en classe. Mais plus que des récits bien organisés, témoignant d'une narration explicative (décrire, c'est raconter pourquoi c'est arrivé), ces différents écrits s'apparentent à un montage de ce qui a plus frappé les élèves. Leur construction relève partiellement d'une recherche de complétude avec des éléments du texte de savoir construit autour de la figure du soldat au combat enrichie de toutes les dimensions vues. Mais ce type de cohérence textuelle n'implique pas pour autant que les élèves se soient vraiment appropriés les notions et les problèmes car elle relève de l'évidence portée par la tradition scolaire et par la littérature à destination des élèves. Elle efface surtout tout souci de rapporter les actions listées aux contextes, où la notion de situation n'aurait pas fonctionné comme déterminisme figé mais où l'explication rassemble les contraintes qui pèsent sur l'action, les conventions qui orientent les choix des acteurs et leurs motivations propres. Ces textes ne sont d'ailleurs soumis à aucun contrôle d'une mise en texte historique.

Ainsi les élèves n'apprennent-ils pas de la situation d'étude de cas de façon magique. Elle échoue à imposer l'intrigue du récit final, sans doute aussi parce que les élèves interprètent la situation à partir de leur propre choix d'éléments. Mais pour comprendre les obstacles dans la double logique cognitive et sociale qui guide notre analyse, la simple transparence des documents, l'effet de plainpied avec le passé ou encore la compréhension de la consigne de rédaction ne suffisent pas. Il nous semble nécessaire de revenir sur les séances précédentes et sur la tension entre les principes pratiques qui guident l'activité des élèves.

\section{Les principes pratiques comme obstacles}

Ces constats d'échec sont à mettre en perspective avec notre développement théorique initial fondé sur la notion de principe pratique. Nous cherchons maintenant à mettre au travail l'hypothèse que ces principes pratiques des élèves constituent des obstacles à l'accès à un savoir problématisé.

\section{L'obstacle du déterminisme}

Une séquence comme celle-ci permet de rendre visibles certains des principes pratiques des élèves de classe d'histoire, notamment à travers les modalités de contrôle des récits produits sur l'expérience de guerre. Pour l'essentiel, le principe mis en évidence est celui d'un déterminisme, qui se rapporte à l'un ou à l'autre des déterminants possibles : soit la cause repose sur le contexte, soit elle renvoie aux volontés individuelles.

C'est en réalité le contexte comme principe explicatif qui domine, notamment par contraste avec les possibilités réduites de mutinerie ou les actions désespérées comme les mutilations ou les désertions. Contexte ou volonté individuelle se répartissent les causes en fonction de l'échelle retenue : pour les élèves, la situation de guerre entre 1914 et 1918 est radicalement contraignante, et les choix ne s'offrent que dans un répertoire réduit et dans des situations pensées comme individuelles. Dans tous les cas, le principe pratique qui contrôle le récit causal se trouve en contradiction avec le principe structurant dont nous avons vu qu'il résultait, historiographiquement parlant, d'un dépassement de cette tension contextes/actions individuelles volontaires qui remet en question les déterminismes.

L'interprétation de la situation de combat comme extrêmement contraignante s'actualise, nous venons de le voir dans l'analyse des textes produits, par la construction de la figure du soldat comme victime. Le principe pratique de la causalité contextuelle joue 
comme obstacle dans la mesure où il intègre systématiquement - sans questionnement - les différents éléments de l'expérience de combat que rencontrent les élèves.

Cependant, le principe pratique déterministe sous sa version contextuelle est renforcé par les quelques éléments qui donnent à penser les causalités par la volonté individuelle. Ainsi en est-il de la situation particulière où les soldats peuvent décider de se mutiler ou de déserter :

\begin{tabular}{|l|l|l|}
\hline 142 & Él & $\begin{array}{l}\text { Ouais ils se blessent pour pouvoir ne } \\
\text { plus aller à la guerre. }\end{array}$ \\
\hline
\end{tabular}

Cette formulation d'un élève (S3A) illustre le pendant de la volonté mais en le confinant à des actions individuelles et qui n'ont pas d'effet sur le déroulement même de la guerre. Ce qui est renforcé par la difficulté que les élèves rencontrent à reprendre la notion de mutinerie qui, dans sa dimension collective, semble hors de portée dans la discussion (dans les deux classes).

On voit bien cette intrication entre contexte et volonté individuelle au bénéfice du premier dans l'ordre des déterminations dans cet échange (S3A) :

\begin{tabular}{|c|c|c|}
\hline 145 & M & $\begin{array}{l}\text { Alors la mutinerie, c'est un petit peu } \\
\text { autre chose, c'est une sorte de révolte. } \\
\text { Des soldats se mettaient plusieurs pour } \\
\text { dire : on refuse d'aller au combat. Ils se } \\
\text { révoltent et ils se mettent à s'opposer } \\
\text { aux ordres des chefs. }\end{array}$ \\
\hline 146 & Él & $\begin{array}{l}\text { Oui mais ils risquaient de se faire } \\
\text { exécuter. }\end{array}$ \\
\hline 147 & M & Oui, bien sûr. \\
\hline 148 & Él & ... il y en avait qui désertait parfois. \\
\hline 149 & M & $\begin{array}{l}\text { Voilà, il y en a qui se mutilait, y en a qui } \\
\text { se mutinait d'accord, [écrit mutinerie] } \\
\text { et puis il y en a aussi qui désertait. C'est } \\
\text { quoi la désertion ? dans l'album d'ail- } \\
\text { leurs il y a un déserteur. }\end{array}$ \\
\hline 150 & Él & $\begin{array}{l}\text { Ben c'est partir en douce, sans se faire } \\
\text { voir. }\end{array}$ \\
\hline 151 & M & Ils se sauvent. \\
\hline 152 & Él & $\begin{array}{l}\text { Mais quand on le récupérait souvent } \\
\text { c'était comme (inaudible). }\end{array}$ \\
\hline 153 & M & $\begin{array}{l}\text { Bien sûr tout ça c'était évidemment, } \\
\text { c'était combattu. Pourquoi est-ce que } \\
\text { les chefs de l'armée étaient très sévères } \\
\text { par rapport à ça, à ces trois choses-là? } \\
\text { Erwan. }\end{array}$ \\
\hline 154 & Erw & $\begin{array}{l}\text { Ben parce qu'ils veulent, ils veulent } \\
\text { gagner la guerre... }\end{array}$ \\
\hline
\end{tabular}

La coercition et les buts de guerre englobent les tentatives souvent infructueuses d'y opposer une réaction individuelle.

Le second objet de discussion où apparaît la volonté individuelle comme cause aux actions est moins évident. Il est d'ailleurs catégorisé spécifiquement dans le travail de la séance 2 : ce qu'il raconte. Bien qu'ainsi distingué, cela correspond à des actions (ce qu'il fait), mais dans un moment particulier de l'expérience combattante : celui de l'attente et de l'intervalle entre les combats effectifs. On a bien là cependant l'expression de choix : les soldats choisissent ce qu'ils souhaitent raconter, à leur famille mais aussi dans leurs journaux.

Dans les deux cas, on constate donc que le principe pratique du déterminisme n'est pas questionné ni remis en cause dans les deux classes. C'est lui qui rend compte des choix faits pour mettre en texte cette expérience de guerre.

\section{Émergence d'un principe pratique méthodologique}

La description précédente se centre sur les principes pratiques que nous avions identifiés au départ et qui relèvent d'une logique théorique. Ils sont construits en référence aux principes structurants proposés par Orange-Ravachol et Beorchia. Mais on peut considérer, dans le cadre de notre hypothèse que ces principes sont construits dans une perspective d'analyse essentiellement épistémologique qui tend à réduire la part du social dans la compréhension des mécanismes de production de savoir. Dans ce cadre problématique, il devient essentiel de poursuivre l'exploration de notre corpus dans un sens socio-cognitif qui observe les différenciations en jeu dans la classe. Nous proposons de le faire sur la base la plus riche du corpus de ce point de vue que constitue la discussion collective de la séance 3.

Le principe pratique d'ordre épistémologique de la causalité directe peut être complété par d'autres types de principes générateurs d'action discernables dans les transcriptions et qui semblent relever davantage de la méthode de travail, et en particulier de l'usage qui est fait des documents de travail disponibles dans la classe, et du rôle accordé à chacun (élèves et maître). Ils génèrent notamment des activités de contrôle, de discussion et de validation des propositions. Tentons de l'appréhender d'abord à travers un extrait significatif (S3A). 


\begin{tabular}{|c|c|c|}
\hline 116 & M & $\begin{array}{l}\text { D'accord, peut-être dans l'action éven- } \\
\text { tuellement, ouais, euh est-ce que } \\
\text { quelqu'un a autre chose à dire par } \\
\text { rapport à ça? la peur du soldat, est-ce } \\
\text { que c'est tout le temps la même? d'ac- } \\
\text { cord, donc là je peux mettre est-ce que } \\
\text { ça change dans le temps. Euh, qui levait } \\
\text { la main, vas-y Marc. }\end{array}$ \\
\hline 117 & Marc & $\begin{array}{l}\text { À mon avis ça change pas parce que... si } \\
\text { ça peut changer parce que s'ils font une } \\
\text { attaque de nuit, et qu'ils doivent tous } \\
\text { sortir des tranchées, là ils ont presque } \\
\text { aucune chance de revoir leur famille. } \\
\text { Donc évidement ils vont penser à eux } \\
\text { [M : d'accord] mais à mon avis ils vont } \\
\text { pas arrêter de penser parce que }\end{array}$ \\
\hline 118 & M & Ouais, je ne sais pas. Oui Zoé vas-y \\
\hline 119 & Zoé & $\begin{array}{l}\text { Oui en fait c'est une question : euh } \\
\text { comment on sait que la nuit les bombar- } \\
\text { dements ils continuaient pas }\end{array}$ \\
\hline 120 & $\mathrm{M}$ & $\begin{array}{l}\text { En fait je ne sais pas concrètement, } \\
\text { il devait y avoir des [... propositions } \\
\text { des él]. Ca reprenait peut-être au petit } \\
\text { matin. Mais il fallait bien qu'ils dorment } \\
\text { quand même. Oui David }\end{array}$ \\
\hline \multicolumn{3}{|l|}{$[\ldots]$} \\
\hline 124 & $\mathrm{M}$ & $\begin{array}{l}\text { Dis-nous un peu plus pourquoi tu } \\
\text { nous dis non [ben non] Est-ce que le } \\
\text { premier combat, est-ce que ça va être } \\
\text { une différence entre la première fois où } \\
\text { il va se retrouver dans cette situation, le } \\
\text { boulanger voilà qui arrive et, est-ce que } \\
\text { tu sais ce qu'il faisait ton arrièrearrière } \\
\text { grand-père comme métier ? un homme } \\
\text { qui dans le civil faisait autre chose et } \\
\text { qui arrive... Eva }\end{array}$ \\
\hline 125 & Eva & $\begin{array}{l}\text { Au début il sait pas trop comment on } \\
\text { fait }\end{array}$ \\
\hline 126 & M & $\begin{array}{l}\text { Au début il sait pas trop, c'est un peu } \\
\text { l'inconnu }\end{array}$ \\
\hline 127 & Eva & $\begin{array}{l}\text { Ben après il a toujours peur mais il } \\
\text { s'habitue }\end{array}$ \\
\hline 128 & M & $\begin{array}{l}\text { Alors au début il sait pas trop, ensuite } \\
\text { malheureusement il sait exactement. } \\
\text { Qu'est-ce que vous en pensez sa peur } \\
\text { elle va changer un peu de nature alors } \\
\text { [oui]. Ouais au début c'est un peu un } \\
\text { trac de quelque chose qu'on connaît } \\
\text { pas, on sait pas comment cela va se } \\
\text { passer. Kevin! tu as des choses à nous } \\
\text { dire là-dessus? Marc? }\end{array}$ \\
\hline 129 & Marc & $\begin{array}{l}\text { En fait j'ai, dans un passage de L'horizon } \\
\text { bleu, ils disaient que [M : l'album ouais] } \\
\text { ils disaient que la nuit était plus dange- } \\
\text { reuse que le jour car pratiquement } \\
\text { toutes les attaques allemandes et fran- } \\
\text { çaises c'était la nuit... }\end{array}$ \\
\hline 130 & $\mathrm{M}$ & J'ai pas compris ça dans l'album \\
\hline 131 & Marc & Oui, un passage... \\
\hline
\end{tabular}

\begin{tabular}{|l|l|l|}
\hline 132 & M & $\begin{array}{l}\text { Tu me remontreras. L'idée c'est que c'est } \\
\text { plus pendant l'hiver et qu'il fait nuit et } \\
\text { c'est sans doute au petit matin. Je pense } \\
\text { qu'en pleine nuit à trois heures du } \\
\text { matin, il n'y avait pas d'attaque, hein. } \\
(\ldots)\end{array}$ \\
\hline
\end{tabular}

Dans cette série d'échanges, isolons ceux qui semblent se répondre directement. À la question de savoir si les soldats ont toujours peur de la même façon, Marc (117) poursuit la discussion initiée par Nicolas en proposant que la pensée dirigée vers la famille est constante chez les soldats, même la nuit, parce que cela dépend surtout de la conscience qu'ils ont du risque qu'ils encourent, accentuée par la répétition des attaques meurtrières qu'ils doivent mener ou subir. Pour expliquer, l'élève s'appuie sur des éléments factuels généraux : attaquer c'est sortir des tranchées et risquer fortement d'y rester, donc ils ne vont pas arrêter d'y penser (à leur famille). Cette proposition est suivie d'une question de Zoé (119) qui porte sur un des éléments factuels (attaques de nuit) affirmé par Marc : " comment on sait que la nuit les bombardements ils continuaient pas? ». Cette question joue le rôle de contrôle du récit explicatif proposé : pour Zoé, et c'est certainement un principe pratique fortement ancré dans la discipline scolaire et son dispositif principal que constitue l'étude de documents, une affirmation en classe d'histoire doit reposer sur des données qu'on trouve dans des documents ou des textes qui font autorité (manuel, maître). L'intervention du maître (120) : bien que soldats, ces êtres humains devaient bien dormir, sousentend que les bombardements ou les attaques n'avaient pas lieu la nuit.

Dans ce contexte d'énonciation, cet élément factuel fait partie d'un récit explicatif commun en construction dans ces échanges. En validant par son autorité - dimension sociale de la situation - le doute de Zoé (119) l'enseignante invalide le récit de Marc (117). Toujours en phase avec le principe pratique d'une validation par les documents, ce dernier va alors chercher dans une des sources disponibles au sein de la classe (l'album L'horizon bleu) un élément en faveur de sa position sur le point contesté. Le choix des mots est essentiel pour nous : «En fait j'ai, dans un passage de L'horizon bleu, ils disaient que [M : l'album ouais] ils disaient que la nuit était plus dangereuse que le jour car pratiquement toutes les attaques allemandes et françaises c'était la nuit...» L'alternance des sujets (je puis ils pour les auteurs) 
montre l'enquête en cours où l'enquêteur cherche du soutien chez les auteurs plus experts de l'album pour évoquer un fait (« en fait») en faveur de son récit.

On aboutit à ce moment-là à une proposition de récit qui est mise en doute sur la base d'une de ses prémisses (il y avait des combats la nuit), par l'intermédiaire des éléments factuels que seules les sources peuvent infirmer ou confirmer. À ce moment-là deux récits d'autorité s'affrontent : le récit direct du maître (120) et le récit de l'album, porté par Marc. La discussion se poursuit et se conclut sur la question de l'interprétation de l'album : "J'ai pas compris ça dans l'album » dit l'enseignante (130) qui renvoie à plus tard («Tu me montreras ») cette question d'interprétation de cette source secondaire.

Cet épisode nous semble heuristique (il est complété par un second du même ordre, que nous présentons après). D'une part il repose sur une mécanique qui montre l'accord dans la classe sur un jeu entre sources et récits explicatifs. Mécanique qui correspond à nombre de résultats de travaux didactiques produits dans le cadre théorique de la discipline scolaire qui rend compte d'un rapport de plain-pied au monde dans l'usage des documents ${ }^{25}$. D'autre part, en insérant ces éléments dans notre problématique, on rend possible la mise en rapport effective de la dimension sociale et de la dimension épistémologique en jeu. Sous la catégorie des principes pratiques, la description précédente donne à voir l'enjeu qui naît de la mise en concurrence dans la classe du récit de l'enseignant et de celui du document. Dans l'activité de construction d'un récit pour la classe, cette concurrence se résout par la hiérarchie des rôles sociaux : Marc comme avocat du document est soumis au récit de l'enseignante. Mais il n'échappe pas à l'observateur que ce type de situation fait émerger un principe de contrôle potentiel, accepté (légitime, convenable) dans la classe : le document est opposable à tout récit. Ordinairement, le dispositif conduit par l'enseignant met les deux en cohérence, mais parfois, comme ici, une divergence apparaît qui conduit au blocage. Dans tous les cas, ce principe pratique méthodologique porte en lui un enjeu toujours déjà social et cognitif.

Selon ce principe qu'on tente d'isoler, les documents ne sont pas considérés comme des récits concurrents des récits en construction, mais plutôt comme des sources au sens littéral du terme : ils font partie de l'explication, ils la composent ; autrement dit il doit y avoir identité des récits. Le récit initial de Marc cite littéralement l'album à propos des « attaques de nuit» pour affirmer que la peur de mourir et de ne plus revoir sa famille ne les quittait pas. Du fait de cette identité incarnée par la citation, les sources doivent, dans le jeu sur lequel s'accordent les élèves et le maître, valider ou invalider le choix du modèle de comportement, mais sans en proposer d'autres : soit elles s'y intègrent, soit le récit est considéré comme faux. Le problème émerge quand il y a difficulté à répondre à une question comme dans cet extrait.

Un second épisode vient compléter cette analyse. Il s'inscrit dans l'évocation des lettres de poilus comme sources et commence par une question sur les actions des soldats (S3A, 201) : «Dans ces lettres, est-ce qu'ils disaient tout? ?. Un élément factuel supposé permet à un élève de répondre par la négative (les enfants lisaient les lettres), ce qui donne un récit explicatif en creux : les soldats s'autocensurent afin de préserver leurs enfants des horreurs de la guerre. Cet élément factuel s'inscrit pour cet élève dans les documents sur René Jacob (référence aux « cadavres d'animaux », 206). Ce récit est donc proposé dans la classe et validé par l'enseignant (207), toujours sur le principe de validation par les sources selon un processus direct et inductif.

C'est l'intervention d'un autre élève qui vient questionner ce processus : Voilà René Jacob, il dit bien, les cadavres [lisant la lettre] "des cadavres allemands, ici, sur le bord de la route, là, dans les ravins et les champs, des cadavres noirâtres, verdâtres, décomposés » (208). Comme dans l'épisode précédemment étudié, le contre-exemple qui conteste le modèle explicatif est rejeté par la maîtresse : "Ouais, ils disaient quand même des choses. [...] Le soldat il voit des choses horribles. Il fait ce qu'on appelle de l'autocensure parce qu'il ne veut pas effrayer, choquer. »(209). Mais la modalité est différente. L'appui sur la source n'est pas contesté parce qu'elle est explicite dans sa contestation de la proposition de réponse du premier élève. Cependant, ce que peut apporter cette source est minoré sans plus d'explication par l'enseignante qui revient à l'écart entre le témoignage et le vécu.

La concurrence entre les deux principes pratiques de validation (par le document, par le professeur) n'est pas explicite dans ce second cas, mais là encore face à la contradiction c'est le maître qui tranche. Cette hiérarchie tend à nier la possibilité d'une interprétation à discuter (ici entre ce qu'il dit de la violence et ce qu'il cacherait). Pour les élèves, 
deux récits d'origine différente peuvent donc cohabiter sans que le choix fait par l'enseignant ne soit raisonné.

On voit par-là que le processus de validation par les documents est largement accepté par les élèves et constitue bien un principe pratique par sa dimension dynamique : il peut générer des prises de position légitimes dans la classe, voire donner aux élèves une position de discussion face au maître. Ce principe semble potentiellement entrer en contradiction avec l'autorité de l'enseignant qui peut trancher contre les prises de positions qui reposent sur lui. Dans cette situation, on peut dire que le jeu est lui-même remis en cause. En outre, ce type de principe se distingue de ceux envisagés jusque-là. Nous proposons de l'appeler principe pratique méthodologique par contraste avec le principe pratique théorique que constitue le déterminisme.

\section{PRINCIPE PRATIQUE THÉORIQUE ET PRINCIPE PRATIQUE MÉTHODOLOGIQUE : EN CLASSE ET CHEZ LES HISTORIENS}

L'introduction de cette distinction par l'analyse de nos données mérite d'être rapportée à ce qui peut se passer chez les historiens afin de penser la pertinence de cette catégorisation. Dans notre corpus, le principe du déterminisme et celui de la validation par les documents semblent d'abord se compléter.

\section{Quels liens entre principes théoriques et méthodologiques?}

On peut tenter dans un premier temps de référer le rapport de ces principes méthodologique et théorique à l'historiographie et l'épistémologie de l'histoire. La relation directe qu'on observe renvoie en première analyse à un positivisme historique qui fait des sources les révélateurs du passé, sources qui parlent d'elles-mêmes et qui ne donnent pas lieu à interprétation. À condition que le travail critique sur ces sources (interne et externe) soit fait selon les règles de la méthode, l'historien s'efface devant ce que les sources ont à dire. Dans la classe observée, ce processus semble fonctionner de manière dégradée ${ }^{26}$. Le travail critique sur les sources n'est que légèrement engagé comme on le voit sur la nature des lettres de poilus à leurs familles; et cette faiblesse du travail critique est compensée par l'autorité de l'enseignant qui coupe court à toute interprétation contradictoire.

Malgré ces différences, le principe méthodologique scolaire conserve une dimension fondamentale de l'approche positiviste : les «faits» sont établis dans les sources, et ils sont la base de la constitution d'un récit qui constitue la réponse attendue ; récit qui résulte donc d'une sélection parmi ces faits et d'une organisation spécifique. Les faiblesses de l'historiographie positiviste sont bien connues et nous donnent les moyens d'interpréter ce qui se produit dans la classe. Les faits ne parlant pas d'eux-mêmes, le rêve objectiviste porté par ce positivisme conduit à masquer les modèles interprétatifs à l'œuvre dans le travail des historiens. Dès lors la question devient celle de la hiérarchie de ces modèles interprétatifs. S'ils ne sont pas discutés dans le travail scientifique, c'est la hiérarchie sociale qui risque de l'emporter dans le travail d'interprétation ainsi dénié. Dans la classe, les élèves n'étant pas vierges de toute idée sur les comportements humains, cette deuxième phase laisse le champ libre au formatage par le principe pratique théorique du déterminisme contextuel ; aucun fait n'est en mesure de le mettre en question.

Ainsi semblent s'articuler les deux types de principes pratiques identifiés : le principe méthodologique de validation par les sources excluant la mise en concurrence des récits et l'interprétation des sources, le principe théorique dominant (le contexte déterminant) devient la seule possibilité de relier les modèles de comportement déjà là dans les conceptions des élèves, et les éléments singuliers apportés par les sources (soit ils s'y insèrent, soit on les récuse) ; comme possibilité unique, ce principe pratique ne peut pas être questionné et la hiérarchie sociale de la classe étant évidente, elle tranche les cas litigieux sans plus de débat.

C'est en cela que l'on peut dire que ce principe théorique est un obstacle dans la classe : il est une solution efficace pour laquelle on ne voit pas d'alternative. Mais il ne se présente ainsi que par la médiation du principe méthodologique positiviste : on ne peut comprendre les logiques cognitives en jeu sans les logiques pratiques afférentes qui dépendent des rôles tenus ordinairement par chacun. Tentons de spécifier ce processus, en le rapportant aux principes méthodologiques qui structurent les pratiques des historiens, dans leurs dimensions sociale et cognitive. 


\section{Dépasser la distinction entre faits et récits}

Comme on le voit, la catégorisation faits/récits est centrale dans l'épistémologie et l'historiographie dans sa version positiviste, mais également dans sa version opposée, incarnée notamment dans le courant du narrative turn (Levisohn, 2002, 2010). Une approche équilibrée rendant compte de la pratique réelle des historiens, repose sur la remise en cause de cette dichotomie, tranche Levisohn.

Dans son analyse épistémologique et historiographique pour penser les enjeux didactiques, il part précisément de la remise en cause de l'objectivité, poussée à son extrême par le courant postmoderne du narrative turn emmené en histoire par l'historien $\mathrm{H}$. White dès les années 1970. Si les faits sont bien établis par une méthode critique, ce qui pose problème dans la prétention à la vérité des historiens se situerait au niveau des récits qui ne sont jamais que les fruits de la « créativité » des historiens ; par-là, l'évaluation des différents récits possibles reposerait sur l'esthétique ou la morale. Pour Levisohn, White inverse ainsi le rapport entre récit et fait des positivistes : les faits sélectionnés pour constituer le corps du récit de l'historien dépendent de l'intrigue choisie, alors que pour le courant positiviste, c'est le récit qui naît des faits avérés. Il parle d'un processus d'impositionnalism, dans le sens où les intrigues imposent leur loi aux faits. Dans cette inversion, les faits ne peuvent pas réfuter les récits.

On voit par ce moyen comment fonctionnent les situations de classe que nous avons décrites. La validation par les faits - très visible dans le travail de la séance 2 - qui structure le travail de la classe avec les documents, loin d'être une validation par le réel qui garantirait une objectivité des récits explicatifs est plutôt une sélection des faits qui s'ignore comme telle. Si l'on reprend la lettre du soldat, on voit par exemple que l'ignorance - dans les écrits comme dans les échanges dans les deux classes - du paradoxe qu'elle exprime (expérience de massacres horribles, mais jugement sur les destructions matérielles comme les pires) peut s'expliquer par le fait qu'il ne s'insère pas dans les modèles explicatifs mis en ouvre dans le cadre du principe pratique du déterminisme contextuel : comme victime, le soldat ne peut penser que la présence de la mort n'est pas le pire. Dans le rapport de validation par les sources, celles-ci irriguant littéralement le récit, la tendance est bien une sélection des faits conformes aux modèles acceptés car dominants ; les faits discordants sont ignorés.

Si la critique postmoderne porte sur les situations de classe envisagées, il est alors essentiel de poursuivre avec l'enquête menée par Levisohn pour envisager épistémologie et pratiques des historiens. Le positivisme comme sa critique radicale repose en fait sur l'évidence d'une dichotomie entre faits et récits qu'il faudrait plutôt contester sur un plan épistémologique. Par le biais d'illustrations, il montre que cette distinction est en fait une différence d'échelle : le fait n'est pas le plus petit commun dénominateur des récits, il est lui-même un récit, mais à une autre échelle. Les conséquences de cette remise en question sont importantes pour nous. C'est le choix de l'échelle qui devient vital pour garantir un rapport des récits aux sources qui vise la recherche de la vérité : ce choix n'est pas le caprice d'un chercheur, il résulte de la rencontre entre les questions du chercheur et les intentions des sources; il rend compte d'une nécessité pratique. Sur le cas imaginaire d'un témoin du franchissement du Rubicon par César, Levisohn montre que le récit n'a d'intérêt pour l'histoire romaine qu'à l'échelle du symbole juridique et politique que constitue cet acte : le témoignage qui ne parle que du franchissement d'une rivière par une armée n'est pas pertinent; il ne le devient que s'il évoque la situation et les règles de la République. $\mathrm{La}$ " perception narrative » des acteurs devient une composante essentielle du rapport du récit aux sources; elle entre en dialogue avec la perception de l'historien. La «mémoire et les attentes » des uns et des autres guident le travail historique. Dès lors, la valeur d'un récit historique ne repose ni directement sur des sources qui ne produiraient que des faits, ni sur les choix moraux ou esthétiques des historiens, mais sur des critères de dialogue entre mémoires et attentes des témoins et acteurs du passé, et mémoires et attentes des historiens d'aujourd'hui.

Dans notre corpus, le jeu de validation par les faits domine largement le travail effectif de la classe. Cela s'observe de manière directe dans la séance 2 qui réclame explicitement de référer les réponses (aux quatre questions : qu'a-t-il vu ? qu'a-t-il fait ? qu'a-t-il ressenti ? qu'a-t-il raconté ?) aux documents. Certains ne font que citer sans construire de récit propre au groupe, comme dans les échanges suivants (S2A; c'est nous qui soulignons) : 


\begin{tabular}{|c|c|c|}
\hline 81 & M & $\begin{array}{l}\text { Alors. Dans le travail de ce groupe, j'au- } \\
\text { rai une critique à faire sur la manière } \\
\text { dont vous avez rempli la consigne. } \\
\text { Est-ce que quelqu'un aurait une } \\
\text { critique à faire sur la manière dont ils } \\
\text { ont rempli la consigne? Marc? }\end{array}$ \\
\hline 82 & Mar & Ils ont surtout recopié des moments \\
\hline 83 & M & $\begin{array}{l}\text { Qu'est-ce qu'ils ont fait, ils ont cité, } \\
\text { ce sont des citations prises dans les } \\
\text { documents. }\end{array}$ \\
\hline 84 & Él & $\begin{array}{l}\text { C'était pas vraiment la consigne. Or la } \\
\text { consigne c'est un peu d'expliquer un } \\
\text { peu et pas de citer les textes. }\end{array}$ \\
\hline 85 & M & $\begin{array}{l}\text { Il fallait interpréter ce qu'on pouvait } \\
\text { lire dans les documents pour dire ce } \\
\text { qu'était l'expérience de RJ. C'est-à-dire } \\
\text { ce qu'il a fait, ce qu'il a vu, etc. donc } \\
\text { là vous avez pas été tout à fait au bout } \\
\text { de la consigne. Allez on continue la } \\
\text { lecture. Lucie. }\end{array}$ \\
\hline
\end{tabular}

L'enseignante explicite ici (85) ce rapport du fait au récit par la notion d'interprétation. Mais, on l'a $\mathrm{vu}$, c'est bien cet intermédiaire de l'interprétation qui est exclu du principe pratique méthodologique dominant. Dans le langage de l'élève (82), la citation renvoie à une action scolaire (recopier) qui n'a pas de valeur épistémologique : on recopie la « bonne» phrase pour répondre à la consigne; il n'y a pas là de place pour l'interprétation.

Plus généralement, la mise en œuvre de ce principe pratique conduit à mener des enquêtes factuelles qui ont la particularité de ne pas renvoyer à des questions historiques, ni de prendre en compte les perceptions des acteurs. On le constate de manière forte dans le flux informel du débat lorsque le hasard des échanges conduit à explorer des liens entre les sources qui permettent d'établir un fait comme dans le cas suivant :

\begin{tabular}{|l|l|l|}
\hline 64 & M & $\begin{array}{l}\text { Les soldats, d'accord. Mais comment } \\
\text { est-ce qu'on peut dire que RJ il était dans } \\
\text { ce groupe-là. Qu'est-ce qui nous permet } \\
\text { de le dire là ? Il n'y a pas son nom quand } \\
\text { même dedans. Qu'est-ce que vous en } \\
\text { pensez les autres, vous êtes d'accord avec } \\
\text { Tom là-dessus? Est-ce qu'on peut dire } \\
\text { que, comme Tom, enfin comme le groupe } \\
\text { de Tom, que ça fait partie de l'expérience } \\
\text { de RJ d'avoir pris la place d'un autre } \\
\text { bataillon et voilà? }\end{array}$ \\
\hline 65 & el & Oui \\
\hline 66 & M & $\begin{array}{l}\text { Oui, pourquoi ? Léo c'est toi qui as dit } \\
\text { oui ? Oui pourquoi alors ? Pourquoi } \\
\text { est-ce qu'on peut dire ça? }\end{array}$ \\
\hline 67 & Léo & Parce que il l'a fait. \\
\hline
\end{tabular}

\begin{tabular}{|l|l|l|}
\hline 68 & M & $\begin{array}{l}\text { Mais il n'y a pas son nom dedans } \\
\text { comment on peut le dire ? }\end{array}$ \\
\hline 69 & Tom & Il y a le titre. \\
\hline 70 & M & $\begin{array}{l}\text { Il y a le titre, ouais. Alors Zoé tu peux } \\
\text { nous le lire. }\end{array}$ \\
\hline 71 & Zoé & $\begin{array}{l}\text { Heu, c'est Journal de l'unité de RJ. Donc } \\
\text { c'est lui qui l'a écrit donc... }\end{array}$ \\
\hline 72 & M & $\begin{array}{l}\text { Ah non. C'est pas, c'est pas lui qui l'a } \\
\text { écrit. Qui a écrit ce journal ? On disait } \\
\text { au début... Paul tu te rappelles ? }\end{array}$ \\
\hline 73 & Paul & \begin{tabular}{l} 
C'est un soldat. \\
\hline
\end{tabular}
\end{tabular}

On a affaire ici à une série d'inductions plutôt qu'à l'élaboration d'un récit explicatif. Il n'a d'ailleurs pas d'autre but que d'explorer en détail les éléments des différents documents étudiés (titre, légende, etc.).

Pourtant, on l'a vu dans l'analyse de la séance 3, il arrive que le récit de l'enseignante ne corresponde pas aux faits constatés par les élèves ou plutôt, comme on le voit bien dans le premier épisode étudié, que le récit de l'enseignante ne corresponde pas au récit de la source telle qu'elle est lue par l'élève. Dans le second épisode, la réponse à la question (est-ce que les lettres disaient tout?) donnée initialement par un des élèves est contredite par l'élève qui cite la lettre de René Jacob, ce qui ne résout pas le problème mais obligerait à approfondir la question : qu'est-ce qu'ils ne disaient pas? Dans ce cas, le récit issu de la lettre s'oppose au récit d'un des élèves, mais sans lui substituer un récit nouveau pour la classe, si ce n'est celui de l'enseignante qui clôt la discussion. Que penser de ces deux situations à la lecture des propositions de Levisohn?

Il semble qu'on peut envisager ces éléments du point de vue de la linéarité du processus de production du récit, selon la perspective dans laquelle Levisohn prend le raisonnement tenu par White. Le récit proposé par un élève est contredit par une source lue par un autre élève, et c'est la maîtresse, par son autorité qui tranche en fonction du modèle explicatif qu'elle choisit. Linéarité qui fait écho à celle du processus décrit par White selon Levisohn : « une fois ces faits réunis, il reste à sélectionner ceux qui sont pertinents et à les organiser $»(2010$, p. 5) ; linéarité qui conduit à l'évitement d'une pratique centrale chez les historiens dont ne rend pas compte l'approche strictement épistémologique de White : la confrontation des récits mène à une pratique de négociation parmi les récits disponibles : «The constructive work of the historical inquirer, then - the creation of historical narratives - is always 
a product of a negotiation among multiple narratives, both "first level" primary-source narratives an "second level" historiographical narratives » (2010, p. 12 ${ }^{27}$. Puisque dans une perspective d'épistémologie pratique la distinction entre faits et récits tend à disparaître, le travail des historiens consiste non plus à construire un récit à l'aide des faits, mais à construire un récit à partir des récits existants, sans que l'un domine par nature l'autre.

D'un point de vue didactique, cette perspective est à la fois plus complexe et plus pertinente. Plus complexe car l'absence de linéarité et de hiérarchie va à l'encontre du principe pratique méthodologique dessiné précédemment ; plus pertinent du fait de la nécessité d'inclure dans le travail de négociation les récits déjà là qu'engendrent les principes pratiques ; et donc de mettre potentiellement ces derniers en question. Cette perspective donne à entrevoir des conditions de remise en question des principes pratiques théoriques par le biais de modification des principes pratiques méthodologiques.

\section{CONCLUSION : QUELLES CONDITIONS POUR FAIRE NÉGOCIER LES ÉLÈVES PARMI LES RÉCITS DISPONIBLES ?}

La séquence forcée, par son échec relatif, constitue un ensemble de données qu'on a pu mettre en tension avec nos questionnements théoriques. La difficulté des deux classes à reconstruire le problème historique plutôt qu'à y appliquer des réponses déjà là peut être décrite dans les termes de la problématisation. Les modèles explicatifs qui actualisent le principe pratique déterministe n'entrent pas en dialogue avec les données parce que celles-ci sont déjà hiérarchisées : ne sont retenues que celles qui confirment le modèle. Lorsqu'il y a un début de tension, nous l'avons vu, la hiérarchie sociale de la classe intervient pour y couper court. Plutôt que d'entrer dans un processus dialectique, comme dans l'espace scientifique étudié par Shinn (1988), la hiérarchie cognitive et la hiérarchie sociale se renforcent l'une l'autre. La robustesse de l'obstacle que constitue le principe pratique en sort accrue, et aucune nécessité n'est produite.

Mais l'analyse à plusieurs échelles de ce cas met en évidence l'intrication inévitable des deux catégories que nous avons séparées pour l'analyse: principes méthodologiques et épistémologiques, savoirs et pratiques, ne fonctionnent qu'ensemble. Fonctionnement qu'on peut alors rapprocher de ce qui se passe dans les champs scientifiques de référence comme le propose notamment Levisohn pour l'histoire. C'est en évitant le face-à-face entre faits et récits qu'on peut envisager les conditions de sortie de cette double hiérarchie figée. Pour y parvenir, il faut substituer le couple traces/récits au couple faits/récits (Levisohn, 2010 ; Seixas \& Peck, 2004 ; Koselleck, 1990) afin de poser, d'une part, l'arbitrage du réel par les traces plutôt que l'arbitrage des faits, et d'autre part la dimension nécessairement sociale (et historique) du texte de savoir comme négociation entre sujets sur la relation sujet/objet que constituent ces processus d'arbitrage des traces. Comment rendre possible ce processus?

Son opérationnalisation passe par la mise en relation d'éléments du passé permettant la construction aujourd'hui du champ des actions possibles dans lequel s'inscrit l'action effective que constitue la source dont on dispose. C'est en resituant ainsi la source qu'on rend raison de son intérêt et de sa valeur pour appréhender le passé.

L'anthropologie y parvient en tentant de sortir du face-à-face entre l'observé et l'ethnographe. Pour F. Weber, c'est le « retour sur les notes ethnographiques $[\ldots]$ qui permet d'y déceler les préjugés qui les sous-tendent et de les rapporter au moment de l'enquête où elles ont été prises. Notes d'enquête et hypothèses progressent les unes en face des autres, se nourrissant mutuellement $»(1996$, p. 360). Les notes de l'ethnographe sur le terrain sont, au même titre que les autres données, prises comme objet d'analyse, selon les préoccupations actuelles de la communauté. Elles sont rapportées au réel des conditions de l'enquête qui prend ensemble les observés et l'ethnographe lui-même. Par ce décentrage, le terrain n'est plus fétichisé, à l'image du travail « de seconde main » qui permet au Mauss de l'essai sur le don de produire « un texte qui élève l'ethnographie à la dignité d'une théorie scientifique » (Weber, 2012). Le terrain n'induit pas l'empirisme, comme la trace en histoire n'induit pas le positivisme.

Ce n'est pas un hasard si Ginzburg se réfère directement au travail anthropologique ainsi recentré (2010a ; 2010b) pour penser l'épistémologie pratique de l'historien. Dans son texte intitulé l'inquisiteur comme anthropologue (2010a, XIV), il analyse rétrospectivement son travail (publié bien avant : 1980) sur le dialogue entre le meunier frioulan $\mathrm{du} \mathrm{XVI}^{\mathrm{e}}$ siècle 
accusé et les inquisiteurs accusateurs, comme rendu possible par le double point d'appui que constituent ces deux protagonistes. Pour comprendre la société de l'Italie du nord de la fin du XVI ${ }^{\text {e }}$ siècle, le cas du meunier Menocchio sous le feu de deux procès de l'Inquisition vaut par son exceptionnalité : il résiste à ses juges, et ces derniers sont intéressés par sa résistance ${ }^{28}$. Cette conjonction fait du corpus documentaire étudié par Ginzburg un ensemble de textes qui tiennent du carnet de terrain de l'ethnographe : les inquisiteurs questionnant le meunier donnent à voir leurs conceptions et, en interaction, celles du meunier. Ce dialogisme des sources (Ginzburg, 2010a) est pour l'historien la condition de son appréhension du champ des actions (des pensées et des discours) possibles d'un homme du peuple, ce que ne permet pas la majorité des dossiers de procès sans résistance des accusés (" monologiques", dit Ginzburg, 2010a, p. 415). Si l'historien ne peut produire aussi facilement que l'ethnographe de telles « notes de terrain », elles lui sont cependant indispensables pour sortir du face-à-face qui tend à instrumentaliser les pratiques étudiées.

Si on s'en tient à notre cas du soldat de la Première guerre, on peut envisager cette condition $\mathrm{du}$ dialogisme des sources pour l'identification du champ des possibles des lettres des Poilus: que pourraient-ils dire qu'ils ne disent habituellement pas ? Par cette identification, il s'agirait de situer les décisions (plus ou moins conscientes) des soldats lorsqu'ils écrivent par rapport à l'expérience qu'ils vivent et qui constitue l'objet même de la recherche en classe. Cette opération passerait par l'usage d'autres sources permettant d'identifier « aussi ce qu'ils pourraient faire mais ne font pas ou du moins pas normalement " (Bazin, 2008, p. 356) : des témoignages rares mais contradictoires (comme un discours d'ancien combattant mis en avant par Prost, relatant le «plaisir de tuer »), les lettres des familles en réponse aux soldats ou les articles de presse indiquant ce que l'arrière pouvait penser - et attendre des soldats du front, et par rapport auxquels répondaient, littéralement, les lettres des soldats. Penser le soldat écrivant, non pas comme un témoin pour nous, mais comme répondant à sa famille, et donc anticipant les attentes de celle-ci, c'est s'engager dans l'explicitation de la valeur de cette lettre pour notre propre enquête. On voit par-là combien « la nécessité d'adopter un point de vue ne concerne pas seulement le témoin, elle s'étend aussi à l'historien ", mais à condition, pour se protéger du relativisme qui guette, de faire porter le travail sur la « résistance intrinsèque des sources [qui] gardent un poids qui leur est propre » (Koselleck, 1990, p. 168-169).

C'est à cette condition que peuvent émerger dans la classe, comme enjeu nouveau, les critères de validation du récit commun par les traces du passé, au sein du processus de "nourrissement mutuel » des notes d'enquête et des hypothèses (des notes de lecture des documents et des explications successives dans la classe d'histoire) dont parle F. Weber, afin d'envisager la remise en cause de la séparation des hiérarchies sociale et cognitive de la classe. 


\section{NOTES}

1. Article qui prolonge un texte auquel nous nous référons également (Orange-Ravachol \& Beorchia, 2007).

2. Dans le cadre théorique de la problématisation, un savoir est problématisé lorsqu'il donne à voir les nécessités qui le fondent : ce n'est pas un savoir que (assertorique), mais c'est savoir pourquoi cela ne peut être autrement (apodictique). Pour une présentation synthétique des enjeux de ce cadre théorique nous renvoyons à l'article déjà cité de Orange-Ravachol et Beorchia (2010), et pour l'histoire en particulier à Doussot (2011).

3. Nous utilisons dans ce texte le jeu comme moyen de rendre compte d'une certaine autonomie de la classe d'histoire en référence aux usages des sciences sociales et en particulier chez Bourdieu (voir notamment Lemieux, 2011, p. 80 : « Jouer est chez Bourdieu, synonyme d'agir car il n'y a pas, selon lui, d'action possible en dehors d'un jeu social »). De ce point de vue, la notion de jeu social est ancrée pour nous sur les mêmes fondements anthropologiques que pour la théorie de l'action conjointe en didactique (Sensevy, 2011).

4. En France mais plus largement, et en particulier en Amérique du Nord.

5. On peut renvoyer à un usage fait dans les sciences de la science de la notion de "pratiques théoriques », comme par exemple Latour dans sa postface à Fleck (2005).

6. « Depuis un siècle, le rapport entre actions humaines et contextes (biologiques, culturels, économiques, etc.) constitue au contraire, pour l'historiographie la plus vivante, un problème ouvert, et non un postulat défini une fois pour toutes dans un sens ou dans l'autre » (Ginzburg, 1997, p. 121).

7. Le lien est direct de la notion de principe pratique à celle d'habitus, comme Bourdieu le précise, par le fait qu'il voulait " mettre en évidence les capacités actives, inventives, "créatrices", de l'habitus et de l'agent (que ne dit pas le terme d'habitude)» (1992, p. 252).

8. Comment expliquer que les paysans acceptent de construire et d'entretenir le château de leur seigneur ?

9. Par cette distinction il semble dépasser la critique formulée par Pestre (2006, p. 32-42) d'une « histoire jugée » à l'aune de la science d'aujourd'hui que dénonce le principe de symétrie de Bloor (la nécessité pour les Science studies de traiter de la même manière la science qui gagne et celle qui échoue). À propos du théorème de Pythagore, Bachelard (1949, p. 96) souligne le " privilège épistémologique » de « l'histoire logique » sur l'histoire du développement du théorème: « l'épistémologie nous enseigne une histoire scientifique telle qu'elle aurait dû être ».
10. « C'est-à-dire qu'ils ne se limitent pas aux solutions du problème mais intègrent également ce qui fait que ce sont ces solutions et pas d'autres qui sont retenues » expliquent Orange-Ravachol et Beorchia (2011).

11. Bourdieu dit : "l'univers des pratiques réellement observées ou potentiellement observables » (1980, p. 170). 12. Sans entrer dans la distinction que développe par exemple Lemieux (2011) entre champ et jeu social, on pourrait penser avec lui que le jeu social spécifique de la science telle qu'elle existe aujourd'hui en fait un champ.

13. Comme le précise Fabiani (2006), la problématique de cette tension essentielle est antérieure de quelques années à la publication de The Structure of Scientific Revolutions (1962).

14. Reproduction déterminée par l'inertie de la discipline scolaire qui n'est certainement pas sans lien avec la réception délicate des travaux didactiques en histoire auprès des enseignants.

15. BO 5 janvier 2012 : « Pouvoir expliquer pourquoi le premier conflit mondial a été appelé "la Grande Guerre" ". BO du 19 juin 2008 : « Le XX siècle et notre époque : La violence du XXe siècle (les deux conflits mondiaux, l'extermination des Juifs et des Tziganes par les nazis, un crime contre l'humanité)».

16. Commémoration aboutissant à une « déshistoricisation de l'Histoire », et fondée sur un processus de victimisation faisant obstacle à la réflexion (Audouin-Rouzeau \& Becker, 2000, p. 8-9).

17. « Corpus de représentations du conflit cristallisé en un véritable système donnant à la guerre sa signification profonde », d'abord défini de façon large pendant et après la guerre, et né en partie avant-guerre, mais que AudouinRouzeau et Becker entendaient d'abord retrouver en rompant avec les reconstructions de l'après-guerre (1997, p. 252).

18. «L'explication de l'histoire et de l'évolution historique par la culture est une erreur comparable à l'ancienne causalité économique. » (Le Goff, 2002, p. 69). Débat que l'on retrouve également chez les anthropologues. Cf. A. Bensa (1996, p. 44) et http://www.vacarme.org/article1604.html $n^{\circ} 44$, été 2008.

19. Cela ressort nettement des justifications données par le GRID lors de la polémique de 2008 portée sur la place publique : "L'histoire de chaque combattant n'est pas simplement une histoire personnelle et singulière : connectée et comparable à celles des autres soldats, elle s'inscrit dans un contexte social qui la détermine fortement. » http://www.laviedesidees.fr/1914-1918-retrouverla-controverse.html.

20. L'analyse de Prost et Winter (2004) en termes de configurations historiographiques nous sert ici d'appui : une configuration historiographique repose sur l'articulation de problèmes, d'argumentations et types de traces. 
Ils expliquent donc que « tous les types d'histoire sont présents au sein de chaque configuration : c'est leur place respective qui se modifie, leur poids qui change, leur rôle dans la problématique et l'argumentation qui se transforme » (p. 49).

21. Dans un cas il s'agit des acteurs en situation de décider et les soldats sont là comme troupes, dans l'autre cas, tous les soldats sont pris en compte dans leur vécu de la guerre. 22. Pour une présentation de cet enjeu, cf. Vézier (2012) et pour un exemple, cf. Vézier (2013) qui étudie la possibilité d'un texte gardant la trace du problème, à partir d'une situation de réécriture en classe de $4^{\mathrm{e}}$.

23. «L'expérience n'est nullement un vécu dont l'historien aurait à rendre compte dans une version singulière et simplifiée qui s'imposerait à lui, tout naturellement, à la seule consultation des faits » (Prochasson, 2008, p. 14).

24. Le corpus est référé en fonction du numéro de la séance (S2) et de la classe concernée (classe A).

25. L'idée de «plain-pied» au monde renvoie aux travaux de O. Orain (2009) en épistémologie et histoire de la géographie. On peut également renvoyer au modèle des 4R de Audigier dans la dimension du « réalisme » qui indique que l'histoire et la géographie « font croire que cette réalité est directement appréhendable et compréhensible moyennant quelques procédures raisonnées » (1995, p. 72). Mais aussi aux études menées ou reprises par Lautier (2003).

26. Comme le suggère la modélisation proposée par Tutiaux-Guillon (2009) à travers le paradigme pédagogique positiviste.

27. «Le travail productif de l'enquêteur en histoire, donc - la création de récits historiques - est toujours le fruit d'une négociation entre plusieurs récits, aussi bien au « niveau premier » des récits-sources, qu'au "niveau deux" des récits historiographiques » (notre traduction).

28. Pour une analyse didactique de cette enquête exemplaire, voir Doussot (2012).

\section{RÉFÉRENCES}

Audigier, F. (1995). Histoire et géographie : des savoirs scolaires en question entre les définitions officielles et les constructions des élèves. Spirale, 15, 61-89.

Audouin-Rouzeau, S. et Becker, A. (2000). 14-18 Retrouver la guerre. Paris: Gallimard.

Audoin-Rouzeau, S. (2009). Controverse ou polémique? La Vie des idées, 5 février 2009.

Audouin-Rouzeau, S. et Becker, A. (1997). Violence et consentement : la « culture de guerre»du premier conflit mondial. In J.-P. Rioux \& J.-F. Sirinelli (dir.), Pour une histoire culturelle (p. 251-271). Paris : Le Seuil.

Bachelard, G. (1949). Le rationalisme appliqué. Paris : Presses universitaires de France.

Bazin, J. (2008). Des clous dans la Joconde. Toulouse : Anacharsis.

Bensa, A. (1996). De la micro-histoire vers une anthropologie critique. In J. Revel (dir.) Jeux d'échelles. La micro-analyse à l'expérience (p. 37-70). Paris : HESS. Gallimard. Le Seuil.

Bourdieu, P. (1972). Esquisse d'une théorie de la pratique. Genève : Droz.

Bourdieu P. (1980). Le sens pratique. Paris : Minuit.

Bourdieu, P. (1992). Les règles de l'art. Genèse et structure du champ littéraire. Paris : Le Seuil.

Bourdieu, P. (2001). Science de la science et réflexivité. Paris : Raisons d'agir.

Buton, F., Loez, A., Mariot, N., Olivera, P. (2008). 19141918 : retrouver la controverse. La Vie des idées, 10 décembre 2008.

Chalak, H. (2012). Problématisation et construction de textes de savoirs dans le domaine du magmatisme au collège. RDST, 6. 119-159.

Delacroix, C. (2010). Acteurs. In C. Delacroix, F. Dosse, P. Garcia \& N. Offenstadt, Historiographies, concepts et débats, (p. 651-663). Paris : Gallimard.

Doussot, S. (2010). Pratiques de savoir en classe et chez les historiens : une étude de cas au collège. Revue française de pédagogie, 173, 85-104.

Doussot, S. (2011). Didactique de l'histoire. Outils et pratiques de l'enquête historienne en classe. Rennes: Presses universitaires de Rennes.

Doussot, S. (2012). Le cas Menocchio et la construction en histoire. Une lecture didactique de l'étude de cas selon Carlo Ginzburg. Le cartable de Clio, 12, 111-125.

Fabiani, J.-L. (2006). À quoi sert la notion de discipline? Enquête, 6, 11-34.

Fabre, M. (2006). Problématisation et débat d'idées : Litiges et différends. Le cas de la controverse de Valladolid. Recherches en éducation, 1, 49-65.

Fabre, M. (2009). Philosophie et pédagogie du problème. Paris : Vrin.

Galvez-Béhar, G. (2009). Le constructivisme de l'historien. Retour sur un texte de Brigitte Gaiti. Le mouvement social, 229, 103-113.

Ginzburg, C. (1980). Le fromage et les vers. Lunivers d'un meunier du $\mathrm{XVI}^{\mathrm{e}}$ siècle. Paris : Flammarion.

Ginzburg, C. (1997). Le juge et l'historien. Considérations en marge du procès Sofri. Paris: Verdier. 
Ginzburg, C. (2001). À distance. Neuf essais sur le point de vue en histoire. Paris: Gallimard.

Ginzburg, C. (2010a). Le fil et les traces. Paris: Verdier.

Ginzburg, C. (2010b). Lectures de Mauss. Annales. Histoire, sciences sociales, 6, 1303-1320.

Grafton, A. (1998). Les origines tragiques de l'érudition. Une histoire de la note de bas de page. Paris : Le Seuil.

Jaubert, M. (2007). Langage et construction de connaissances à l'école. Un exemple en sciences. Bordeaux : Presses universitaires de Bordeaux.

Jaubert, M., Rebière, M., Bernié, J.-P. (2003). Lhypothèse « communauté discursive » : d'où vient-elle ? où va-telle? Les cahiers Théodile, 4, 51-80.

Koselleck, R. (1990). Le futur passé. Contribution à la sémantique des temps historiques. Paris : éditions de l'EHESS.

Koselleck, R. (1997). L'expérience de l'histoire. Paris : Gallimard, Le Seuil.

Kuhn, T. (1990). La tension essentielle : tradition et changement dans les sciences. Paris : Gallimard.

Latour, B. (1989). La science en action. Paris : La découverte.

Latour, B. (2005). Transmettre la syphilis. Partager l'objectivité. Postface à Fleck, L. Genèse et développement d'un fait scientifique. Paris : Flammarion.

Lautier, N. (1997). À la rencontre de l'histoire. Villeneuve d'Ascq : Presses universitaires du Septentrion.

Lautier, N. (2003). Histoire enseignée, histoire appropriée. Quelques éléments spécifiques d'une didactique de l'histoire. In N. Tutiaux-Guillon, A. Bruter et M.-C. Baquès. Pistes didactiques et chemin d'historien. Textes offerts à H. Moniot (p. 357-379). Paris : LHarmattan.

Le Goff, J. (2002). L'Histoire. In Université de tous les savoirs. L'histoire, la sociologie et l'anthropologie, volume 2 (p. 59-75). Paris : Odile Jacob.

Lemieux, C. (2011). Le crépuscule des champs. Limites d'un concept ou disparition d'une réalité historique? Raisons pratiques, 21, 75-100.

Le Marec, Y. et Vézier, A. (2006). Comment les soldats de la Grande Guerre ont-ils tenu ? Un débat d'histoire dans la classe. Le cartable de Clio, 6, 160-173.

Le Marec, Y., Doussot, S., Vézier, A. (2009). Savoir, problèmes et pratiques langagières en histoire. Éducation E Didactique, 3, 3, 7-28.

Levisohn, J. (2002). Stories about Stories about History Hayden White, Historiography, and History Education. Philosophy of education, 465-472.

Levisohn, J. (2010). Negotiating Historical Narratives: An Epistemology of History for History Education. Journal of Philosophy Education, 44, 1, 1-21.

Orain, O. (2009). De plain-pied dans le monde, Écriture et réalisme dans la géographie française au $X X^{e}$ siècle. Paris : L'Harmattan.

Orange, C. (2002). Apprentissages scientifiques et problématisation. Les sciences de l'éducation, pour l'ère nouvelle, 35, 1, 25-42.

Orange, C. (2005). Problématisation et conceptualisation en sciences et dans les apprentissages scientifiques. Les sciences de l'éducation - Pour l'ère nouvelle, 38, 3, 69-93.
Orange, C. (2010). Situations forcées, recherches didactiques, et développement du métier enseignant. Recherches en éducation, HS, 2, 73-85.

Orange-Ravachol, D. et Beorchia, F. (2007). Principes structurants et savoirs en sciences de la vie et de la Terre. Congrès international AREF.

Orange-Ravachol, D. et Beorchia, F. (2011). Principes structurants et construction de savoirs en sciences de la vie et de la Terre. Éducation E Didactique, 5, 1, 7-28.

Pestre, D. (2006). Introduction aux Science Studies. Paris : La Découverte.

Prochasson, C. (2008). 14-18. Retours d'expériences. Paris: Texto.

Prost, A. (2006). Comment a évolué l'histoire de la Grande Guerre. Le Cartable de Clio, 6, 11-21.

Prost, A. et Winter, J. (2004). Penser la Grande Guerre. Un essai d'historiographie, Paris: Le Seuil.

Revel, J. (1996). Jeux d'échelles. La micro-analyse de l'expérience. Paris: Gallimard-Le Seuil.

Revel, J. (2009). Le pied du diable. Sur les formes de la cumulativité en histoire. Enquête, 8, 85-110.

Rey, B. (2002). Diffusion des savoirs et textualité. Recherche et Formation, 40, 43-57.

Ricœur, P. (1983). Temps et récit. T. 1 L’intrigue et le récit historique. Paris : Le Seuil.

Risbjerg Eskildsen, K. (2007). Leopold von Ranke, la passion de la critique et le séminaire d'histoire. In C. Jacob (dir.), Lieux de savoir (vol. 1) : espaces et communautés (p. 462-482). Paris: Albin Michel.

Seixas, P. et Peck, C. (2004). Teaching historical thinking. In A. Sears \& I. Wright (Eds.), Challenges and Prospects for Canadian Social Studies (pp. 109-117). Vancouver: Pacific Educational Press.

Sensevy, G. (2011). Le sens du savoir. Éléments pour une théorie de l'action conjointe en didactique. Bruxelles: De Boeck.

Shinn, T. et Ragouet, P. (1988). Hiérarchies des chercheurs et formes des recherches. Actes de la recherche en sciences sociales, 74, 2-22.

Shinn, T. (2005). Controverses sur la science. Pour une sociologie de l'activité scientifique. Paris : Raisons d'agir.

Tutiaux-Guillon, N. (2009). L'histoire scolaire française entre deux modèles: contenus, pratiques et finalités. Revue française d'éducation comparée, 4, 105-116.

Vézier, A. (2012). La leçon de Braudel, récit et problème en histoire. Le Cartable de Clio, 12, 99-110.

Vézier, A. (2013). L'apprentissage par problématisation, un nouveau modèle pour interroger la textualité des savoirs historiques scolaires ? Recherches en didactiques, 16, 11-28.

Weber, F. (1996). De la modélisation à la description armée : le cas de l'ethnographie réflexive. In J.-Y. Grenier, C. Grignon \& Menger P.-M. (dir.), Le modèle et le récit (p. 355-364). Paris : éditions de la Maison des sciences de l'homme.

Weber, F. (2012). Vers une ethnographie des prestations de marché. In M. Mauss, Essai sur le don (p. 1-55). Paris : PUF. 